\title{
Diversidad y distribución de la tribu Vernonieae (Asteraceae) en México
}

\section{Diversity and distribution of the tribe Vernonieae (Asteraceae) in Mexico}

\author{
Rosario Redonda-Martínez ${ }^{1,2 *}$ (1)
}

1 Universidad Nacional Autónoma de México, Posgrado en Ciencias Biológicas, Avenida Ciudad Universitaria 3000, O4360 Coyoacán, Cd. Mx., México.

2 Adscripción actual: Instituto de Ecología, A.C., Centro Regional del Bajío, Avenida Lázaro Cárdenas 253, 61600 Pátzcuaro, Michoacán, México.

*mrrm.unam@yahoo.com.mx

\section{Citar como:}

Redonda-Martínez, R. 2017. Diversidad y distribución de la tribu Vernonieae (Asteraceae) en México. Acta Botanica Mexicana 119: 115-138. DOI: http://dx.doi.org/10.21829/ abml19.2017.1235

Recibido: 6 de marzo de 2016 Revisado: 13 de octubre de 2016 Aceptado: 23 de noviembre de 2016.

DOI:

http://dx.doi.org/10.21829/abm119.2017.1235

\section{Resumen:}

Antecedentes y Objetivos: La tribu Vernonieae se distribuye principalmente en trópicos y subtrópicos de ambos hemisferios e incluye 21 subtribus, 118 géneros y alrededor de 1050 especies. Los objetivos de este trabajo son proporcionar una lista actualizada de las especies mexicanas de la tribu y aportar datos referentes a su distribución por estado, altitud, tipos de vegetación, hábito de crecimiento y fenología.

Métodos: Se realizó una revisión bibliográfica de diversos trabajos taxonómicos y colecciones científicas de México y algunas del extranjero.

Resultados clave: Se reconocen 73 especies y dos taxones infraespecíficos pertencientes a 19 géneros que se agrupan en ocho subtribus. De los 19 géneros, tres son endémicos y tres introducidos; 47 especies y dos taxones infraespecíficos son endémicos y tres introducidos. Chiapas, Oaxaca y Veracruz concentran el mayor número de géneros y especies, además de Guerrero. En el bosque de Pinus-Quercus se encuentra la mayor diversidad de especies de Vernonieae, las cuales se distribuyen desde el nivel del mar hasta los $3000 \mathrm{~m}$ de altitud.

Conclusiones: Más de $60 \%$ de las especies de Vernonieae presentes en el país son endémicas y únicamente $4.2 \%$ corresponde a taxones introducidos.

Palabras clave: Compositae, endemismo, rareza, sinonimia.

\section{ABSTRACT:}

Background and Aims: The tribe Vernonieae is distributed in the tropics and subtropics of both hemispheres and includes 21 subtribes, 118 genera and about 1050 species. The aims of this work are to provide an updated list of Mexican species of the tribe and contribute data concerning their distribution by state, altitude, vegetation types, growth habit and phenology.

Methods: A bibliographic review of several taxonomic works and scientific collections of Mexico and some foreign ones was carried out.

Key results: Seventy-tree species and two infraspecific taxa belonging to 19 genera are recognized, grouped in eight subtribes. Of these 19 genera, three are endemic and three are introduced, 47 species and two infraspecific taxa are endemic and three species are introduced. Chiapas, Oaxaca and Veracruz concentrate the largest number of genera and species, in addition to Guerrero. In the PinusQuercus forest can be encountered the largest diversity of Vernonieae species, which are distributed from sea level to $3000 \mathrm{~m}$ altitude.

Conclusions: More than $60 \%$ species of Vernonieae presents in country are endemic and only $4.2 \%$ correspond to introduced taxa.

Key words: Compositae, endemism, rarity, synonymy.

\section{INTRODUCCIÓN}

La tribu Vernonieae presenta cabezuelas homógamas discoides, flores actinomorfas generalmente moradas, a veces rosadas o blancas, estilos delgados con las ramas agudas, pilosas a lo largo de toda su extensión y polen equinado y triaperturado (Fig. 1). Es un grupo monofilético relacionado con Lactuceae y Liabeae, siendo 


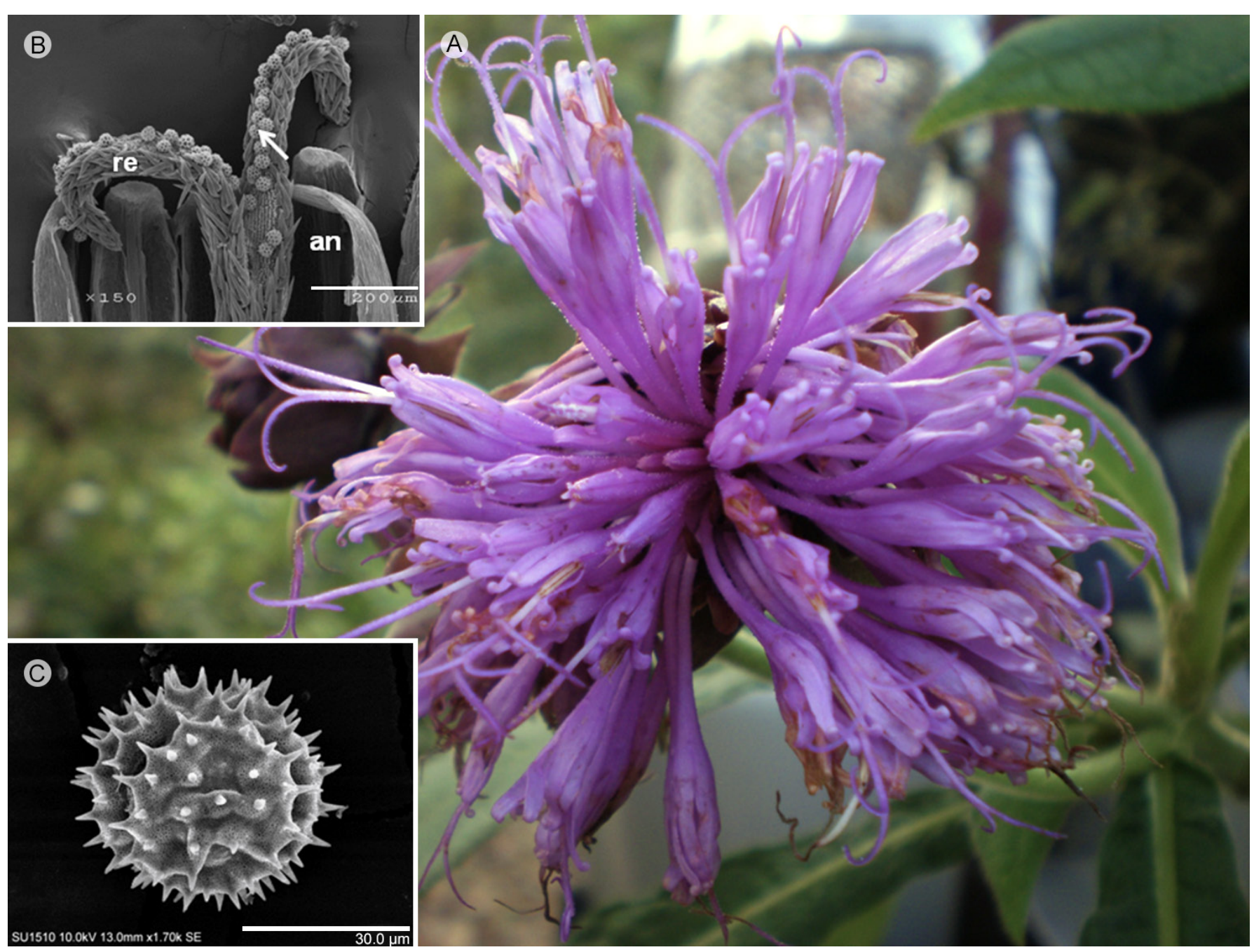

Figura 1: Características distintivas de Vernonieae. A. cabezuela de Lepidonia callilepis (Gleason) H. Rob. \& V.A. Funk; B. flor de Elephantopus mollis Kunth, mostrando las ramas del estilo pilosas, re: ramas del estilo, an: antera, la flecha señala los granos de polen; C. polen de L. callilepis con ornamentación equinada. Fotografías A. Rosario Redonda-Martínez; B.-C. Berenit Mendoza-Garfías.

esta última su grupo hermano (Bremer, 1987; Keeley y Turner, 1990; Jansen et al., 1991; Keeley y Jansen, 1994; Kim y Jansen, 1995; Panero y Funk, 2002, 2008; Funk et al., 2005, 2009; Robinson, 2007; Robinson y Funk, 2011).

La delimitación tribal de Vernonieae se ha mantenido prácticamente sin cambios desde que fue propuesta por Cassini (1819). Sin embargo, en jerarquías subtribales y genéricas, la tribu ha presentado diversos problemas taxonómicos, debido principalmente al gran número de especies agrupadas en el género Vernonia Schreb., el cual hasta finales de la década de 1970, incluía 1000-1500 es- pecies (Jones, 1977). Bajo este concepto Vernonia presentaba una enorme variación morfológica, la cual derivó en la descripción de una gran cantidad de especies, muchas de ellas actualmente reducidas a sinonimia. Los otros géneros reconocidos eran monotípicos o incluían menos de 10 especies. Bentham (1873) propusó una de las primeras clasificaciones infratribales con base en el arreglo de las cabezuelas, reconociendo dos subtribus: Euvernonieae y Lychnophoreae. En la primera subtribu incluyó a los taxones con cabezuelas separadas y en la segunda a los taxones con cabezuelas agregadas. Más de un siglo después, 
Jones (1979a, 1981) enfocó su atención en Vernonia s.l., utilizando caracteres morfológicos, químicos, citológicos y palinológicos; reconoció dos subgéneros: Vernonia con distribución americana y Orbisvestus S.B. Jones, con distribución en el Viejo Mundo. Asimismo, dividió a cada subgénero en un gran número de secciones, subsecciones y series, las cuales lejos de resolver los problemas taxonómicos derivaron en una clasificación artificial. Sin embargo, en trabajos posteriores, realizados tanto con caracteres morfológicos (Keeley y Turner, 1990), como moleculares (Keeley y Jansen, 1994; Keeley et al., 2007), los dos subgéneros identificados por Jones se han mantenido como linajes independientes.

La propuesta de clasificación más reciente de la tribu (Keeley y Robinson, 2009) se fundamenta en los resultados de estudios filogenéticos, así como trabajos taxonómicos tradicionales en los que se han esclarecido los límites genéricos en complejos de especies de Vernonia s.l., el restablecimiento de varios géneros reducidos a sinonimia y la propuesta de nuevos géneros (Robinson, 1980, 1987a, 1987b, 1990, 1992, 1993). En esta propuesta se reconocen 21 subtribus, 118 géneros y alrededor de 1050 especies.

Vernonieae se distribuye en trópicos y subtrópicos de ambos hemiferios; en América su distribución abarca desde el sur de Canadá hasta el centro de Argentina; está ausente en el centro y sur de los Andes, la meseta baja del Amazonas y el oeste de Estados Unidos de América. Se ha propuesto que la tribu se originó en los trópicos porque en estas regiones se encuentran las especies ancestrales y los principales centros de diversidad, uno en América al sur de Brasil y otro en África (Jones, 1977, 1979a, 1981; Keeely y Turner, 1990; Keeley y Jansen, 1994).

Los objetivos de este estudio son proporcionar una lista actualizada de las especies mexicanas de Vernonieae y aportar datos referentes a su distribución por estado, altitud, tipos de vegetación, hábito de crecimiento y fenología.

\section{MATERIALES Y MÉTODOS}

Se realizó una revisión minuciosa de diversos trabajos florístico-taxonómicos de la tribu Vernonieae (Gleason,
1906, 1922, 1923; Robinson y Reed, 1973; Jones, 1973; 1976, 1977, 1979b; King y Jones, 1975; Chapman y Jones, 1978; Jones y Stutts, 1981; McVaugh, 1984; Redonda-Martínez, 2006, 2016; Redonda-Martínez y Villaseñor, 2009, 2011, 2013, 2014; Redonda-Martínez y MartínezSalas, 2015; Redonda-Martínez et al., 2016b; Robinson, 1980, 1987a, 1987b, 1990, 1992, 1993, 1999; Robinson y Funk, 1987; Turner, 1981, 1988, 1992, 1997, 2007; Rzedowski y Calderón de Rzedowski, 1995), para generar un listado de las especies presentes en México. Asimismo, se revisaron ejemplares herborizados de Vernonieae, depositados en diversas colecciones científicas del país (CIB, CIIDIR, ENCB, FCME, IEB, MEXU, XAL, XALU) y el extranjero (CTES, F, MO, TEX, US), con la finalidad de cotejar el listado obtenido de la revisión bibliográfica con los ejemplares de herbario. Con base en los datos de las etiquetas de los ejemplares herborizados, se obtuvo información de fenología, distribución por estado y tipos de vegetación nombrados de acuerdo con la clasificación de Rzedowski (1978).

\section{Resultados}

\section{Diversidad}

En México la tribu Vernonieae está representada por ocho subtribus y 19 géneros, 73 especies y dos categorías infraespecíficas. Del total de los géneros, tres son endémicos: Bolanosa A. Gray, Leiboldia Schltdl. y Stramentopappus H. Rob. \& V.A. Funk, $\mathrm{y}$ tres, Cyanthillium Blume, Centratherum Cass. y Struchium P. Browne, son introducidos. Seis se han segregado de Vernonia s.1. (Fig. 2): Critoniopsis Sch. Bip., Leiboldia, Lepidaploa (Cass.) Cass., Lepidonia S.F. Blake, Stramentopappus y Vernonanthura H. Rob. (Villaseñor et al., 1998). El género más diverso es Critoniopsis con 23 especies, seguido de Vernonia s.s. con 17 y dos categorías infraespecíficas, mientras que Bolanosa es monotípico. Del total de especies, 47 y dos categorías infraespecíficas son endémicas y tres introducidas. Las 23 especies restantes extienden su distribución hacía Estados Unidos de América, las 

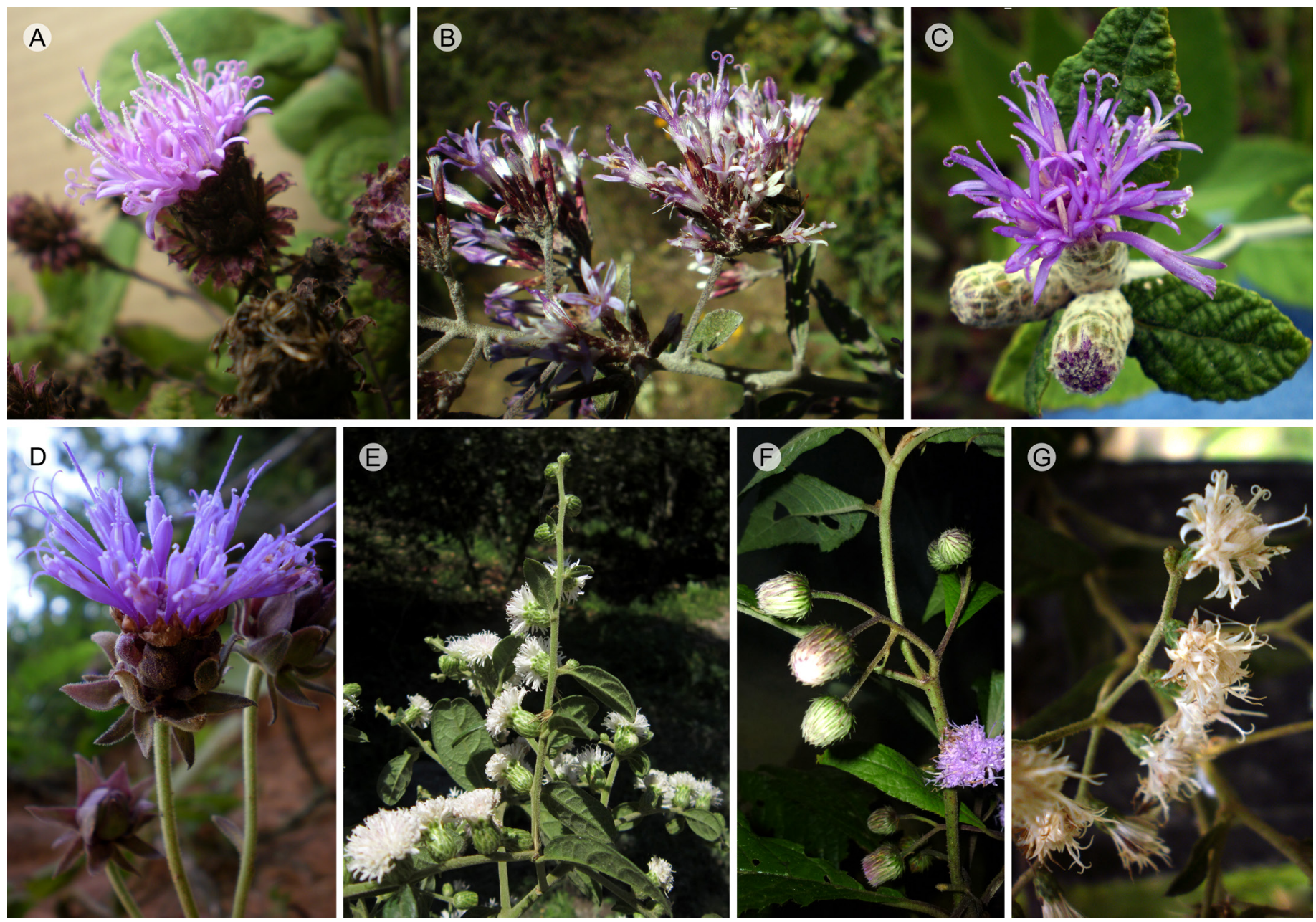

Figura 2: Géneros segregados de Vernonia Schreb., y especies mexicanas representativas de Vernonieae. A. Vernonia karvinskiana DC. subsp. inuloides (DC.) S.B. Jones, B. Critoniopsis tomentosa (La Llave \& Lex.) H. Rob., C. Leiboldia guerreroana (S.B. Jones) H. Rob., D. Lepidonia callilepis (Gleason) H. Rob. \& V.A. Funk, E. Stramentopappus pooleae (B.L. Turner) H. Rob. \& V.A. Funk, F. Lepidaploa tortuosa (L.) H. Rob., G. Vernonanthura patens (Kunth) H. Rob. Fotografías A-D, G. Rosario Redonda-Martínez; E. Esteban Martínez-Salas; F. Jesús López-Santiago.

Antillas, Centro y Sudamérica, y el Viejo Mundo; tres de ellas se comparten con el sur de Estados Unidos, cinco con Centroamérica, ocho con Centro y Sudamérica y cinco con Estados Unidos de América, Centro y Sudamérica y el Viejo Mundo, incluyendo en esta última categoría a las especies introducidas.

A continuación se presenta la lista de especies reconocidas y el nomenclátor taxonómico de la tribu Vernonieae en México. Los taxones se agrupan en subtribus de acuerdo con la clasificación de Keeley y Robinson (2009). Las subtribus y especies están enumeradas de manera consecutiva, con números romanos y arábigos, res- pectivamente. Los nombres válidos de cada especie están resaltados en negritas; las especies endémicas están precedidas por un asterisco $(*)$ y las introducidas por el signo más $(+)$, los nombres que carecen de símbolo corresponden a taxones nativos de México pero con distribución en otras regiones del mundo. Los sinónimos homotípicos están precedidos por el signo $(\equiv)$ y los heterotípicos por el símbolo de (=).

\section{Subtribu Centratherinae}

1. $\quad$ Centratherum punctatum Cass., Dict. Sci. Nat. 7: 384.1817. 
$=$ Centratherum intermedium (Link) Less., Linnaea 4: 320.1829.

$=$ Centratherum muticum (Kunth) Less., Linnaea 4: 320. 1829.

EBaccharoides punctatum (Cass.) Kuntze, Rev. Gen. P1., 1: 320. 1891.

=Baccharoides muticum (Kunth) Kuntze, Rev. Gen. P1., 1: 320. 1891.

\section{Subtribu Erlangeinae}

2. $\quad$ Cyanthillium cinereum (L.) H. Rob., Proc. Biol. Soc. Wash. 103: 252. 1990.

三Vernonia cinerea (L.) Less., Linnaea 4: 291. 1829.

$\equiv$ Cacalia cinerea (L.) Kuntze, Rev. Gen. Pl. 1: 323. 1891.

\section{Subtribu Elephantopinae}

3. Elephantopus mollis Kunth, Nov. Gen. Sp., folio ed. 4: 20.1818.

=Elephantopus hypomalacus S.F. Blake, Contr. Gray Herb. 52: 20. 1917.

4. Orthopappus angustifolius (Sw.) Gleason, Bull. New York Bot. Gard. 4: 238. 1906.

$\equiv$ Elephantoppus angustifolius Sw., Prodr. 115. 1788.

5. Pseudelephantopus spicatus (Juss. ex Aubl.) Rohr, Skr. Naturh.-Selsk. Kjob. 2: 214. 1792.

$\equiv$ Elephantopus spicatus Juss. ex Aubl., Hist. P1. Guiane 2: 808. 1775.

\section{Subtribu Leiboldiinae}

6. *Bolanosa coulteri A. Gray, Smithsonian Contr. Knowl. 3: 82. 1852.

$\equiv$ Vernonia coulteri (A. Gray) B.L. Turner, Brittonia 33: 405. 1981.

=Vernonia floccosa M.E. Jones, Contr. West. Bot. 18: 69. 1933. non Gardn. 1846.

7. *Leiboldia guerreroana (S.B. Jones) H. Rob., Phytologia 87: 82. 2005.

三Vernonia guerreroana S.B. Jones, Castanea 44: 233. 1979.
8. *Leiboldia serrata (D. Don) Gleason, Bull. New York Bot. Gard. 4: 164. 1906.

$\equiv$ Diazeuxis serrata D. Don, Trans. Linn. Soc. 16: 254. 1830.

=Vernonia arctioides Less., Linnaea 6: 400. 1831.

$=$ Leiboldia arctioides (Less.) Schltdl., Linnaea 19:

743. 1847.

=Vernonia leiboldiana Schltdl., Linnaea 19: 742 . 1847.

$=$ Cacalia leiboldiana (Schltdl.) Kuntze, Rev. Gen. P1. 2: 970. 1891.

=Leiboldia leiboldiana (Schltd1.) Gleason, Bull. New York Bot. Gard. 4: 163. 1906.

9. *Lepidonia alba Redonda-Mart. \& E. Martínez, Syst. Bot. 40: 1139. 2015.

10. *Lepidonia callilepis (Gleason) H. Rob. \& V.A. Funk, Bot. Jahrb. Syst. 108: 225. 1987.

三Vernonia callilepis Gleason, N. Amer. F1. 33: 91. 1922.

11. *Lepidonia jonesii (B.L. Turner) H. Rob. \& V.A. Funk, Bot. Jahrb. Syst. 108: 225. 1987.

EVernonia jonesii B.L. Turner, Brittonia 33: 408.

12. *Lepidonia mexicana (Less.) H. Rob. \& V.A. Funk, Bot. Jahrb. Syst. 108: 225. 1987.

三Vernonia mexicana Less., Linnaea 6: 680. 1831.

ECacalia mexicana (Less.) Kuntze, Rev. Gen. Pl. 2: 970.1891.

$\equiv$ Leiboldia mexicana (Less.) Gleason, Bull. New York Bot. Gard. 4: 163. 1906.

13. Lepidonia salvinae (Hemsl.) H. Rob. \& V.A. Funk, Bot. Jahrb. Syst. 108: 225. 1987.

$\equiv$ Vernonia salvinae Hemsl., Biol. Centr.-Amer. Bot. 2: 73. 1881.

$\equiv$ Cacalia salvinae (Hemsl.) Kuntze, Rev. Gen. Pl. 2: 970. 1891.

$\equiv$ Leiboldia salvinae (Hemsl.) Gleason, Bull. New York Bot. Gard. 4: 162. 1906.

14. *Lepidonia wendtiana (B.L. Turner) RedondaMart. \& Villaseñor, Rev. Mex. Biodivers. 84: 984. 2013. 
三Vernonia wendtiana B.L. Turner, Phytologia 82: 275. 1997.

15. *Stramentopappus congestiflorus Redonda-Mart. \& Villaseñor, Rev. Mex. Biodivers. 85: 2. 2014.

16. *Stramentopappus pooleae (B.L. Turner) H. Rob. \& V.A. Funk, Bot. Jahrb. Syst. 108: 227. 1987.

$\equiv$ Vernonia pooleae B.L. Turner, Brittonia 33: 406. 1981.

\section{Subtribu Lepidaploinae}

17. Harleya oxylepis (Benth.) S.F. Blake, J. Wash. Acad. Sci. 22: 381. 1932.

$\equiv$ Oliganthes oxylepis Benth., Gen. P1. 2: 233. 1873.

18. *Lepidaploa boquerona (B.L. Turner) H. Rob., Smithsonian Contr. Bot. 89: 70. 1999.

三Vernonia boquerona B.L. Turner, Phytologia 81: 115. 1996.

19. Lepidaploa canescens (Kunth) H. Rob., Proc. Biol. Soc. Wash. 103: 481. 1990.

$\equiv$ Vernonia canescens Kunth, Nov. Gen. Sp. (folio) 4: 27.1818.

=Vernonia hirsutivena Gleason, Bull. New York Bot. Gard. 4: 175. 1906.

= Vernonia purpusii Brandegee, Univ. Calif. Publ. Bot. 6: 197. 1915.

= Vernonia cuneifolia (Britton) Gleason, Amer. J. Bot. 10: 301. 1923, non Gardn., 1846.

$\equiv$ Cacalia canescens (Kunth) Kuntze, Rev. Gen. Pl. 2: 969.1891.

=Vernonia rusbyi Gleason, Amer. J. Bot. 19: 753. 1932.

20. *Lepidaploa diazlunana (B.L. Turner) H. Rob., Smithsonian Contr. Bot. 89: 71. 1999.

$\equiv$ Vernonia diazlunana B.L. Turner, Phytologia 69: 169. 1990.

21. *Lepidaploa koelzii (McVaugh) H. Rob., Smithsonian Contr. Bot. 89: 71. 1999.

$\equiv$ Vernonia koelzii McVaugh, Contr. Univ. Mich. Herb. 9: 480. 1972.

22. Lepidaploa polypleura (S.F. Blake) H. Rob., Smithsonian Contr. Bot. 89: 72. 1999. $\equiv$ Vernonia polypleura S.F. Blake, J. Wash. Acad. Sci. 28: 478. 1938.

23. Lepidaploa remotiflora (Rich.) H. Rob., Proc. Biol. Soc. Wash. 103: 491. 1990.

$\equiv$ Vernonia remotiflora Rich., Actes Soc. Hist. Nat. Paris. 1: 112. 1792.

=Vernonia acilepis Benth. ex Orsted, Vidensk. Meddel. Dansk Naturhist. Foren. Kjob. 1852: 68. 1853 .

$\equiv$ Cacalia remotiflora (Rich.) Kuntze, Rev. Gen. Pl. 2: 970.1891.

24. Lepidaploa salzmanii (DC.) H. Rob., Proc. Biol. Soc. Wash. 103: 493. 1990.

三Vernonia salzmannii DC., Prodr. 5: 55. 1836.

=Vernonia poeppigiana DC., Prodr. 5: 55. 1836, non DC., Prodr. 5: 20. 1836.

=Vernonia argyropappa $\mathrm{H}$. Buek, Gen. Sp. Synon. Cand. 2: 5.1840.

= Cacalia argyropappa (H. Buek) Kuntze, Rev. Gen. P1. 2: 969. 1891.

$\equiv$ Cacalia salzmannii (DC.) Kuntze, Rev. Gen. Pl. 2: 971.1891.

=Cacalia poeppigiana (DC.) Kuntze, Rev. Gen. Pl. 2: 971.1891.

25. Lepidaploa tortuosa (L.) H. Rob., Proc. Biol. Soc. Wash. 103: 495. 1990.

EConyza tortuosa L., Sp. P1. 2: 862. 1753.

=Conyza scandens Mill., Gard. Dict. (ed. 8). 11. 1768.

=Vernonia schiedeana Less., Linnaea 6: 399. 1831.

=Vernonia seemanniana Steetz, Bot. Voy. Herald 139. 1854.

= Cacalia schiedeana (Less.) Kuntze, Rev. Gen. Pl. 2: 971.1891.

=Cacalia seemanniana (Steetz) Kuntze, Rev. Gen. P1. 2: 971. 1891.

$=$ Vernonia vernicosa Klatt, Bull. Soc. Roy. Bot. Belgique. 35: 294. 1896.

=Vernonia vernicosa Klatt var. comosa Greenm., Proc. Amer. Acad. Arts. 39: 98. 1903. 
三Vernonia tortuosa (L.) S.F. Blake, Proc. Biol. Soc. Wash. 39: 144. 1926.

26. Lepidaploa uniflora (Mill.) H. Rob., Proc. Biol. Soc. Wash. 103: 496. 1990.

EConyza uniflora Mill., Gard. Dict. (ed. 8). 13. 1768.

ECacalia uniflora (Mill.) Kuntze, Rev. Gen. Pl. 2: 969. 1891.

=Vernonia ctenophora Gleason, Bull. Torrey Bot. Club. 46: 243. 1919.

27. Stenocephalum jucundum (Gleason) H. Rob., Proc. Biol. Soc. Wash. 100: 583. 1987.

EVernonia jucunda Gleason, Bull. Torrey Bot. Club. 46: 248. 1919.

28. +Struchium sparganophorum (L.) Kuntze, Rev. Gen. P1. 1: 366. 1891.

Ethulia sparganophora L., Sp. Pl. ed. 2, 1171. 1763.

ESparganophorus sparganophora (L.) C. Jeffrey, Kew Bull. 43: 272. 1988. tautónimo illeg.

\section{Subtribu Piptocaphinae}

29. *Critoniopsis autumnalis (McVaugh) H. Rob., Proc. Biol. Soc. Wash. 106: 609. 1993.

$\equiv$ Vernonia autumnalis McVaugh, Contr. Univ. Mich. Herb. 9: 477. 1972.

30. *Critoniopsis baadii (McVaugh) H. Rob., Proc. Biol. Soc. Wash. 106: 609. 1993.

$\equiv$ Critoniopsis salicifolia var. baadii McVaugh, Contr. Univ. Mich. Herb. 9: 484. 1972.

$\equiv$ Vernonia baadii (McVaugh) S.B. Jones, Brittonia 25: 113. 1973.

31. *Critoniopsis barbinervis (Sch. Bip.) H. Rob., Proc. Biol. Soc. Wash. 106: 609. 1993.

$\equiv$ Vernonia barbinervis Sch. Bip. in Seem., Bot. Voy. Herald 297. 1856.

ECacalia barbinervis (Sch. Bip.) Kuntze, Rev. Gen. P1. 2: 969. 1891.

$\equiv$ Eremosis barbinervis (Sch. Bip.) Gleason, Bull. New York Bot. Gard. 4: 232. 1906.
32. *Critoniopsis feddemae (McVaugh) Villaseñor \& Redonda-Mart., Rev. Mex. Biodivers. 84: 983. 2013.

三Vernonia feddemae McVaugh, Contr. Univ. Mich. Herb. 9: 480. 1972.

33. *Critoniopsis foliosa (Benth.) H. Rob., Proc. Biol. Soc. Wash. 106: 614. 1993.

=Monosis foliosa Benth., Pl. Hartw. 19. 1839.

=Vernonia steetzii Sch. Bip. in Seem., Bot. Voy. Herald 297. 1856.

=Vernonia steetzii var. callilepis Sch. Bip. in Seem., Bot. Voy. Herald 297. 1856.

$\equiv$ Vernonia foliosa (Benth.) Sch. Bip., Jahresber. Pollichia 18/19: 161. 1891, non Gardn. 1846.

= Cacalia steetzii (Sch. Bip.) Kuntze, Rev. Gen. Pl. 2: 971.1891.

$\equiv$ Eremosis foliosa (Benth.) Gleason, Bull. New York Bot. Gard. 4: 228. 1906.

$=$ Eremosis steetzii (Sch. Bip.) Gleason, Bull. New York Bot. Gard. 4: 230. 1906.

=Vernonia mucronata S.F. Blake, Contr. Gray Herb. 52: 19. 1917.

=Eremosis callilepis (Sch. Bip.) Gleason, N. Amer. F1. 33: 98. 1922.

=Vernonia aristifera S.F. Blake, Contr. U.S. Natl. Herb. 23: 1415. 1926.

=Vernonia steetzii var. aristifera (S.F. Blake) McVaugh, Contr. Univ. Mich. Herb. 9: 484. 1972.

34. *Critoniopsis heydeana (J.M. Coult.) H. Rob., Proc. Biol. Soc. Wash. 106: 614. 1993.

EVernonia heydeana J.M. Coult., Bot. Gaz. (Crawfordsville) 20: 42.1895.

$\equiv$ Eremosis heydeana (J.M. Coult) Gleason, Bull. New York Bot. Gard. 4: 234. 1906.

35. Critoniopsis leiocarpa (DC.) H. Rob., Proc. Biol. Soc. Wash. 106: 615. 1993.

三Vernonia leiocarpa DC., Prodr. 5: 34. 1836.

$\equiv$ Cacalia leiocarpa (DC.) Kuntze, Rev. Gen. P1. 2: 970. 1891.

EEremosis leiocarpa (DC.) Gleason, Bull. New York Bot. Gard. 4: 232. 1906. 
=Eremosis melanocarpa Gleason, Bull. New York Bot. Gard. 4: 232. 1906.

=Vernonia melanocarpa (Gleason) S.F. Blake, Contr. Gray Herb. 52: 18. 1917.

36. *Critoniopsis littoralis (Brandegee) H. Rob., Proc. Biol. Soc. Wash. 106: 616. 1993.

EVernonia littoralis Brandegee, Erythea 7: 3. 1899. $\equiv$ Eremosis littoralis (Brandegee) Gleason, N. Amer. Fl. 33: 100. 1922.

37. *Critoniopsis macphersonii (S.B. Jones \& Stutts) H. Rob., Proc. Biol. Soc. Wash. 106: 616. 1993. $\equiv$ Vernonia macphersonii S.B. Jones \& Stutts, Brittonia 33: 546. 1981.

38. *Critoniopsis macvaughii (S.B. Jones) H. Rob., Proc. Biol. Soc. Wash. 106: 616. 1993.

$\equiv$ Vernonia macvaughii S.B. Jones, Brittonia 25: 105. 1973.

39. *Critoniopsis obtusa (Gleason) H. Rob., Proc. Biol. Soc. Wash. 106: 617. 1993.

$\equiv$ Eremosis obtusa Gleason, N. Amer. Fl. 33: 99. 1922.

$\equiv$ Vernonia obtusa (Gleason) S.F. Blake, Contr. U.S. Natl. Herb. 23: 1415. 1926.

=Vernonia obtusa subsp. parkeri S.B. Jones, Brittonia 25: 108. 1973.

=Vernonia duncanii S.B. Jones, Brittonia 25: 108. 1973.

=Vernonia duncanii subsp. bartlettii S.B. Jones, Brittonia 25: 110. 1973

= Vernonia obtusa var. bartlettii (S.B. Jones) B.L. Turner, Phytologia 66: 462. 1989.

=Vernonia obtusa var. parkeri (S.B. Jones) B.L. Turner, Phytologia 66: 462. 1989.

=Critoniopsis duncanii (S.B. Jones) H. Rob., Proc. Biol. Soc. Wash. 106: 612. 1993.

40. *Critoniopsis oolepis (S.F. Blake) H. Rob., Proc. Biol. Soc. Wash. 106: 617. 1993.

$\equiv$ Vernonia oolepis S.F. Blake, Contr. Gray Herb. 52: 20. 1917.

$\equiv$ Eremosis oolepis (S.F. Blake) Gleason, N. Amer. F1. 33: 97.1922.
41. *Critoniopsis ovata (Gleason) H. Rob., Proc. Biol. Soc. Wash. 106: 617. 1993.

$\equiv$ Eremosis ovata Gleason, Bull. Torrey Bot. Club 40: 331. 1913.

=Vernonia durangensis S.F. Blake, Contr. U.S. Natl. Herb. 22: 587. 1924.

=Vernonia gleasonii S.F. Blake, Contr. Gray Herb. 52: 17. 1917. non Ekman, 1914.

42. *Critoniopsis pallens (Sch. Bip.) H. Rob., Proc. Biol. Soc. Wash. 106: 619. 1993.

$\equiv$ Vernonia pallens Sch. Bip., Jahresber. Pollichia 18/19: 161. 1891.

$\equiv$ Eremosis pallens (Sch. Bip.) Gleason, Bull. New York Bot. Gard. 4: 228. 1906.

=Vernonia michoacana McVaugh, Contr. Univ. Mich. Herb. 9: 482. 1972.

43. *Critoniopsis pugana (S.B. Jones \& Stutts) H. Rob., Proc. Biol. Soc. Wash. 106: 620. 1993.

$\equiv$ Vernonia pugana S.B. Jones \& Stutts, Brittonia 33: 544. 1981.

44. *Critoniopsis salicifolia (DC.) H. Rob., Proc. Biol. Soc. Wash. 106: 621. 1993.

=Monosis salicifolia DC., Prodr. 5: 77. 1836.

$\equiv$ Vernonia salicifolia (DC.) Sch. Bip., Linnaea 20: 507. 1847, non Less. 1829, nec Gillies ex Hook. \& Arn., 1835.

$\equiv$ Cacalia salicifolia (DC.) Kuntze, Rev. Gen. P1. 2: 971. 1891.

=Eremosis leiophylla Gleason, Bull. New York Bot. Gard. 4: 231. 1906.

$\equiv$ Eremosis salicifolia (DC.) Gleason, Bull. New York Bot. Gard. 4: 231. 1906.

=Vernonia leiophylla (Gleason) S.F. Blake, Contr. Gray Herb. 52: 18. 1917.

45. Critoniopsis shannonii (J.M. Coult.) H. Rob., Proc. Biol. Soc. Wash. 106: 621. 1993.

三Vernonia shannonii J.M. Coult., Bot. Gaz. (Crawsfordville) 20: 42. 1895.

$\equiv$ Eremosis shanonii (J.M. Coult.) Gleason, Bull. New York Bot. Gard. 4: 234. 1906. 
46. *Critoniopsis solorzanoana (Rzed. \& Calderón) H. Rob., Smithsonian Contr. Bot. 89: 64. 1999. $\equiv$ Vernonia solorzanoana Rzed. \& Calderón, Acta Bot. Mex. 32: 7. 1995.

47. *Critoniopsis tarchonanthifolia (DC.) H. Rob., Proc. Biol. Soc. Wash. 106: 624. 1993.

$\equiv$ Monosis tarchonanthifolia DC., Prodr. 5: 77. 1836.

= Oliganthes kanvinskii Sch. Bip., Linnaea 20: 505. 1847.

$\equiv$ Vernonia tarchonanthifolia (DC.) Sch. Bip., Linnaea 20: 507. 1847.

=Vernonia purpurascens Sch. Bip. in Walp., Rep. Bot. Syst. 2: 945.1843.

ECacalia tarchonanthifolia (DC.) Kuntze, Rev. Gen. P1. 2: 271. 1891.

= Cacalia karwinskii (Sch. Bip.) Kuntze, Rev. Gen. P1. 2: 270. 1891.

$\equiv$ Eremosis tarchonanthifolia (DC.) Gleason, Bull. New York Bot. Gard. 4: 230. 1906.

48. *Critoniopsis tomentosa (La Llave \& Lex.) H. Rob., Proc. Biol. Soc. Wash. 106: 624. 1993.

$\equiv$ Turpinia tomentosa La Llave \& Lex., Nov. Veg. Descr. 1: 24. 1824.

=Vernonia paniculata DC., Prodr. 5: 23. 1836.

$\equiv$ Monosis tomentosa (La Llave \& Lex.) DC., Prodr. 5: 77.1836.

=Vernonia purpurascens Sch. Bip., Repert. Bot. Syst. 2: 945.1843.

=Vernonia monosis Sch. Bip., Linnaea 20: 507. 1847.

ECacalia tomentosa (La Llave \& Lex.) Kuntze, Rev. Gen. P1. 2: 969. 1891.

=Cacalia monosis (Sch. Bip.) Kuntze, Rev. Gen. P1. 2: 970. 1891.

= Cacalia paniculata (DC.) Kuntze, Rev. Gen. Pl. 2: 970.1891.

$\equiv$ Eremosis tomentosa (La Llave \& Lex.) Gleason, Bull. New York Bot. Gard. 4: 229. 1906.

=Eremosis purpurascens (Sch. Bip.) Gleason, Bull. New York Bot. Gard. 4: 233. 1906.
49. Critoniopsis triflosculosa (Kunth) H. Rob., Proc. Biol. Soc. Wash. 106: 624. 1993.

$\equiv$ Vernonia triflosculosa Kunth, Nov. Gen. Sp. (folio) 4: 40. 1818 .

=Vernonia triantha Nees \& Schauer, Linnaea 19: 714. 1847.

$\equiv$ Cacalia triflosculosa (Kunth) Kuntze, Rev. Gen. P1. 2: 971. 1891.

= Cacalia triantha (Nees \& Schauer) Kuntze, Rev. Gen. Pl. 2: 971.1891.

=Vernonia palmeri Rose, Contr. U.S. Natl. Herb. 1: 101. 1891.

$=$ Eremosis palmeri (Rose) Gleason, Bull. New York Bot. Gard. 4: 233. 1906.

$\equiv$ Eremosis triflosculosa (Kunth) Gleason, Bull. New York Bot. Gard. 4: 233. 1906.

=Vernonia chacalana S.F. Blake, Contr. Gray Herb. 52: 19. 1917.

=Vernonia triflosculosa subsp. palmeri (Rose) S.B. Jones, Brittonia 25: 100. 1973.

=Vernonia triflosculosa var. palmeri (S.B. Jones) B.L. Turner, Phytologia 66: 464. 1989.

50. *Critoniopsis uniflora (Sch. Bip.) H. Rob., Proc. Biol. Soc. Wash. 106: 625. 1993.

三Vernonia uniflora Sch. Bip., Linnaea 20: 506. 1847.

=Eupatorium uniflorum Sessé \& Moç., Pl. Nov. Hisp. 164. 1890.

$\equiv$ Cacalia uniflora (Sch. Bip.) Kuntze, Rev. Gen. P1. 2: 968. 1891.

$=$ Cacalia baptizanda Kuntze, Rev. Gen. Pl. 2: 968. 1891.

51. *Critoniopsis villaregalis (Carvajal) H. Rob., Smithsonian Contr. Bot. 89: 65. 1999.

EVernonia villaregalis Carvajal, Phytologia 49: 193. 1981.

=Vernonia tequilana S.B. Jones \& Stutts, Brittonia 33: 544. 1981.

$=$ Critoniopsis tequilana (S.B. Jones \& Stutts) H. Rob., Proc. Biol. Soc. Wash. 106: 624. 1993. 
52. Piptocarpha poeppigiana (DC.) Baker, F1. Bras. 6: 131.1873.

三Vernonia poeppigiana DC. Prodr. 5: 20. 1836.

=Piptocarpha chontalensis Baker, F1. Bras. 6: 132. 1873.

\section{Subtribu Rolandrinae}

53. Spiracantha cornifolia Kunth, Nov. Gen. Sp. (folio) $4: 27.1818$.

\section{Subtribu Vernoniinae}

54. Cyrtocymura scorpioides (Lam.) H. Rob., Proc. Biol. Soc. Wash. 100: 852. 1987.

$\equiv$ Conyza scorpioides Lam., Encycl. Méthod. 238. 1786.

$\equiv$ Vernonia scorpioides (Lam.) Pers., Syn. Plant. 2: 404. 1807.

$\equiv$ Lepidaploa scorpioides (Lam.) Cass., Dict. Sci. Nat. 26: 16. 1823, comb. inval., previa a la publicación válida del género Lepidaploa.

$\equiv$ Cacalia scorpioides (Lam.) Kuntze, Rev. Gen. P1. 2: 971. 1891.

55. Vernonanthura cordata (Kunth) H. Rob., Phytologia 73: 70. 1992.

$\equiv$ Vernonia cordata Kunth, Nov. Gen. Sp. (folio) 4 : 31. 1818 .

$\equiv$ Cacalia cordata (Kunth) Kuntze, Rev. Gen. Pl. 2: 969. 1891.

=Vernonia morelana Gleason, Bull. Torrey Bot. Club 46: 241. 1919.

=Vernonia cordata var. hooveri McVaugh, Contr. Univ. Mich. Herb. 9: 479. 1972.

56. Vernonanthura patens (Kunth) H. Rob., Phytologia 73: 72. 1992.

$\equiv$ Vernonia patens Kunth, Nov. Gen. Sp. (folio) 4: 32. 1818.

=Vernonia deppeana Less., Linnaea 6: 398. 1831.

=Vernonia haenkeana DC., Prodr. 5: 37. 1836.

=Vernonia aschenborniana Schauer, Linnaea 19:

714. 1847.

= Cacalia deppeana (Less.) Kuntze, Rev. Gen. Pl. 2: 970.1891.
=Vernonia viarum M.E. Jones, Contr. West. Bot. 18: 69. 1933.

=Vernonanthura deppeana (Less.) H. Rob., Phytologia 73: 70. 1992.

57. *Vernonia alamanii DC., Prodr. 5: 61. 1836.

ECacalia alamanii (DC.) Kuntze, Rev. Gen. Pl. 2: 969. 1891.

=Vernonia dictyophlebia Gleason, Bull. New York Bot. Gard. 4: 203. 1906.

=Vernonia alamanii var. dictyophlebia (Gleason) McVaugh, Contr. Univ. Mich. Herb. 9: 477. 1972.

58. *Vernonia barclayi H. Rob. \& C.F. Reed, Phytologia 27: 52. 1973.

59. *Vernonia bealliae McVaugh, Contr. Univ. Mich. Herb. 9: 477. 1972.

=Vernonia corymbiformis Gleason, Bull. New York Bot. Gard. 4: 199. 1906, non DC. 1836.

60. *Vernonia boolleana Sch. Bip., Bot. Voy. Herald 297. 1856.

61. *Vernonia confusa Redonda-Mart., Villaseñor \& A. Campos, Phytotaxa. 282: 211. 2016.

62. *Vernonia cronquistii S.B. Jones, Rhodora 78: 194. 1976.

$\equiv$ Vernonanthura cronquistii (S.B. Jones) H. Rob., Phytologia 73: 70. 1992.

63. *Vernonia faustiana (C.G. Chapm. \& S.B. Jones) B.L. Turner, Phytologia 65: 136. 1988.

三Vernonia greggii A. Gray subsp. faustiana G.C. Chapm. \& S.B. Jones, Sida 7: 280. 1978.

64. *Vernonia greggii A. Gray, Proc. Amer. Acad. Arts 17: 204. 1882.

=Vernonia ervendbergii A. Gray, Proc. Amer. Acad. Arts 17: 203. 1882.

=Vernonia taylorae Standl., Field Mus. Publ. Bot. 17: 224. 1937.

=Vernonia schaffneri A. Gray, Proc. Amer. Acad. Arts 17: 204. 1882.

=Vernonia greggii var. palmeri A. Gray, Proc. Amer. Acad. Arts 17: 204. 1882.

=Vernonia greggii subsp. ervendbergii (A. Gray) G.C. Chapm. \& S.B. Jones, Sida 7: 279. 1978. 
=Vernonia greggii subsp. schaffneri (A. Gray) G.C. Chapm. \& S.B. Jones, 7: 279. 1978.

65. *Vernonia hintoniorum B.L. Turner, Phytologia 77: 408. 1994.

EVernonanthura hintoniorum (B.L. Turner) H. Rob., Smithsonian Contr. Bot. 89: 85. 1999.

66. *Vernonia joyaliae B.L. Turner, Phytologia 73: 16. 1992.

67a. *Vernonia karvinskiana DC. subsp. karvinskiana, Prodr. 5: 62. 1836.

=Vernonia corymbiformis DC., Prodr. 5: 62. 1836.

=Cacalia corymbiformis (DC.) Kuntze, Rev. Gen. P1. 2: 969. 1891.

ECacalia karvinskiana (DC.) Kuntze, Rev. Gen. P1. 2: 970. 1891.

=Vernonia conzatii B.L. Rob., Proc. Amer. Acad. 44: 615. 1909.

67b. *Vernonia karvinskiana subsp. inuloides (DC.)

S.B. Jones, Rhodora 78: 188. 1976.

$\equiv$ Vernonia inuloides DC., Prodr. 5: 62. 1836.

$\equiv$ Cacalia inuloides (DC.) Kuntze, Rev. Gen. Pl. 2: 970. 1891.

68. Vernonia larsenii B.L. King \& S.B. Jones, Brittonia 27: 84. 1975.

$\equiv$ Vernonia lindheimeri var. leucophylla Larsen, Ann. Missouri Bot. Gard. 15: 333. 1928.

69. *Vernonia liatroides DC., Prodr. 5: 34. 1836.

=Vernonia ehrenbergiana Sch. Bip., Linnaea 20: 513. 1847.

=Eupatorium tulanum Klatt, Abh. Naturf. Ges. Halle 15: 323. 1882.

$\equiv$ Cacalia liatroides (DC.) Kuntze, Rev. Gen. P1. 2: 971. 1891.

= Cacalia ehrenbergiana (Sch. Bip.) Kuntze, Rev. Gen. P1. 2: 971. 1891.

=Vernonia capraefolia Gleason, Bull. New York Bot. Gard. 4: 200. 1906.

=Vernonia liatroides subsp. ehrenbergiana (Sch. Bip.) S.B. Jones, Rhodora 78: 192. 1976.

=Vernonia liatroides subsp. gentryi S.B. Jones, Rhodora 78: 193. 1976.
= Vernonanthura liatroides (DC.) H. Rob., Phytologia 73: 71. 1992.

70. Vernonia lindheimeri A. Gray \& Engelm., Proc. Amer. Acad. Arts 1: 46. 1846.

ECacalia lindheimeri (A. Gray \& Engelm.) Kuntze, Rev. Gen. P1. 2: 970. 1891.

71. Vernonia marginata (Torr.) Raf., Atl. J. 146. 1832. EVernonia altissima var. marginata Torr., Ann. Lyc. New York 2: 210. 1827.

=Vernonia jamesii Torr. \& A. Gray, Fl. N. Amer. 2: 58. 1841.

ECacalia marginata (Torr.) Kuntze, Rev. Gen. P1. 2: 968.1891.

=Vernonia tenuifolia Small, Bull. Torrey Bot. Club 25. 145. 1898.

72. Vernonia oaxacana Sch. Bip. ex Klatt, Leopoldina 20: 74.1894.

$\equiv$ Vernonanthura oaxacana (Sch. Bip. ex Klatt) H. Rob., Phytologia 73: 72. 1992.

73. *Vernonia serratuloides Kunth, Nov. Gen. Sp. (folio) 4: 33. 1818.

=Vernonia sinclairii Benth., Bot. Voy. Sulphur 109. 1845.

=Perezia paniculata A. Gray, Proc. Amer. Acad. Arts 21: 393. 1886.

=Perezia vernonioides A. Gray, Proc. Amer. Acad. Arts 22: 433. 1887.

$\equiv$ Cacalia serratuloides (Kunth) Kuntze, Rev. Gen. P1. 2: 970. 1891.

= Cacalia sinclairii (Benth.) Kuntze, Rev. Gen. Pl. 2: 970.1891.

=Vernonia jaliscana Gleason, Bull. New York Bot. Gard. 4: 198. 1906.

= Vernonia umbellifera Gleason, Bull. New York Bot. Gard. 4: 199. 1906.

=Vernonia vernonioides (A. Gray) Bacigalupi, Contr. Gray Herb. 97: 77. 1931.

=Vernonia camporum M.E. Jones, Contr. West. Bot. 18: 69. 1933.

=Vernonia serratuloides Kunth, subsp. vernonioides (A. Gray) S.B. Jones, Rhodora 78: 200. 1976. 
= Vernonanthura sinclairii (Benth.) H. Rob., Phytologia 73: 73. 1992.

$\equiv$ Vernonanthura serratuloides (Kunth) H. Rob., Phytologia 73: 73. 1992.

\section{Especie excluida}

Lepidaploa arborescens (L.) H. Rob., Proc. Biol. Soc. Wash. 103: 481. 1990.

\section{Distribución geográfica}

Chiapas, Oaxaca y Veracruz concentran el mayor número de géneros y especies, además de Guerrero. En primer lugar se encuentra Oaxaca con 34 especies y dos categorías infraespecíficas, en segundo lugar están Chiapas y Guerrero con 23; en tercero, Veracruz con 22 taxones; mientras que en la península de Baja California, la tribu está ausente (Cuadro 1). Las especies de Vernonieae se distribuyen desde el nivel del mar hasta los $3000 \mathrm{~m}$ de altitud.

\section{Distribución por tipos de vegetación}

Los bosques de Pinus-Quercus concentran la mayor cantidad de especies con 43 y dos categorías infraespecíficas. En segundo lugar se encuentran los bosques de Quercus, con 40 especies y dos categorías infraespecíficas y en tercero los bosques mesófilos de montaña con 35 especies.

En el Cuadro 2 se desglosa la distribución por estado, tipos de vegetación y rango de altitud en el que se han colectado cada una de las especies reconocidas. En el cuadro 3 se incluyen datos de fenología y forma de vida de cada una de las especies.

\section{Formas de vida y fenología}

La mayoría de las especies de Vernonieae son arbustos o hierbas perennes, también se encuentran algunos árboles y trepadoras; solamente un taxon presenta hábito herbáceo (C. cinereum). La mayor parte de las especies presentan periodos de floración y fructificación de unos cuantos meses; sin embargo, seis taxones florecen y fructifican durante todo el año (Cuadro 3).
Cuadro 1: Diversidad de géneros y especies de Vernonieae, por estado. Los números en negritas corresponden al total de géneros y especies (incluyendo nativos, endémicos e introducidos), los números entre paréntesis representan los géneros o especies endémicos de un estado y los números entre corchetes los taxones introducidos. En la primera columna entre paréntesis se encuentran las abreviaturas de cada uno de los estados, las cuales se retoman en el cuadro 2.

\begin{tabular}{lcc}
\hline Estado & Géneros & Especies \\
\hline Aguascalientes (Ags.) & $\mathbf{1}$ & $\mathbf{2}$ \\
Campeche (Camp.) & $\mathbf{6}$ & $\mathbf{8}[1]$ \\
Chihuahua (Chih.) & $\mathbf{2}$ & $\mathbf{4}$ \\
Chiapas (Chis.) & $\mathbf{1 5}[3]$ & $\mathbf{2 3}(2)[3]$ \\
Ciudad de México (Cd. Mx.) & $\mathbf{1}$ & $\mathbf{1}$ \\
Coahuila (Coah.) & $\mathbf{1}$ & $\mathbf{5}(1)$ \\
Colima (Col.) & $\mathbf{5}$ & $\mathbf{1 0}(1)$ \\
Durango (Dgo.) & $\mathbf{3}$ & $\mathbf{1 0}$ \\
Guanajuato (Gto.) & $\mathbf{3}$ & $\mathbf{7}$ \\
Guerrero (Gro.) & $\mathbf{9}$ & $\mathbf{2 3}(1)[1]$ \\
Hidalgo (Hgo.) & $\mathbf{6}$ & $\mathbf{1 0}$ \\
Jalisco (Jal.) & $\mathbf{8}$ & $\mathbf{2 0}(4)$ \\
México (Méx.) & $\mathbf{5}$ & $\mathbf{9}$ \\
Michoacán (Mich.) & $\mathbf{5}$ & $\mathbf{1 4}(1)$ \\
Morelos (Mor.) & $\mathbf{4}$ & $\mathbf{8}$ \\
Nayarit (Nay) & $\mathbf{1 3}[2]$ & $\mathbf{2 2}[2]$ \\
Nuevo León (N.L.) & $\mathbf{8}[1]$ & $\mathbf{9}[1]$ \\
Oaxaca (Oax.) & $\mathbf{3}$ & $\mathbf{9}$ \\
Puebla (Pue.) & $\mathbf{7}$ & $\mathbf{1 3}(1)$ \\
Querétaro (Qro.) & $\mathbf{2}$ & $\mathbf{2}$ \\
Quintana Roo (Q. Roo) & $\mathbf{1 4}(1)[2]$ & $\mathbf{3 4}(6)[2]$ \\
San Luis Potosí (S.L.P.) & $\mathbf{9}[1]$ & $\mathbf{1 4}[1]$ \\
Sinaloa (Sin.) & $\mathbf{5}$ & $\mathbf{1 2}$ \\
Sonora (Son.) & $\mathbf{7}[1]$ \\
Tabasco (Tab.) & $\mathbf{3}$ & $\mathbf{1 1}$ \\
Tamaulipas (Tam.) & $\mathbf{8}$ \\
Veracruz (Ver.) & $\mathbf{3}(2)$ \\
Yucatán (Yuc.) & $\mathbf{1 1}[1]$ \\
Zacatecas (Zac.) & $(1)[2]$ \\
\hline & &
\end{tabular}


Cuadro 2: Distribución por estado, tipos de vegetación e intervalos altitudinales de especies de la tribu Vernonieae presentes en México. Las especies endémicas están precedidas por un asterisco y las introducidas por el signo +. Los tipos de vegetación se abrevian de la siguiente manera: bosque de Abies (BA), bosque de galería (BG), bosque mesófilo de montaña (BMM), bosque de Juniperus (BJ), bosque de Pinus (BP), bosque de Pinus-Quercus (BPQ), bosque de Quercus (BQ), bosque tropical perennifolio (BTP), bosque tropical caducifolio (BTC), bosque tropical subcaducifolio (BTSC), manglar (MAN), matorral xerófilo (MX), palmar (PAL), pastizal incluyendo sabana (PAZ), cultivada (CU) y vegetación secundaria (VS). El rango de altitud está dado en metros sobre el nivel del mar.

\begin{tabular}{|c|c|c|c|c|}
\hline Subtribu & Especie & Distribución por estado & Tipo de vegetación & Altitud \\
\hline Centratherinae & + Centratherum punctatum Cass. & $\begin{array}{l}\text { Chis., Gro., Oax., Pue., Tab., Ver., Centro } \\
\text { a Sudamérica, Antillas, Asia, Australia }\end{array}$ & CU, VS & $20-1450$ \\
\hline Erlangeinae & + Cyanthillium cinereum (L.) H. Rob. & $\begin{array}{l}\text { Camp., Chis., Oax., Q. Roo, Tab., Ver., } \\
\text { Yuc., Centro a Sudamérica, Antillas, } \\
\text { África, Asia, Australia }\end{array}$ & VS & $0-400$ \\
\hline \multirow[t]{3}{*}{ Elephantopinae } & Elephantopus mollis Kunth & $\begin{array}{l}\text { Chis., Col., Gro., Jal., Méx., Nay., Oax., } \\
\text { Pue., Qro., S.L.P., Sin., Tab., Tam., Ver., } \\
\text { Estados Unidos de América, Centro } \\
\text { a Sudamérica, Antillas, África, Asia, } \\
\text { Australia }\end{array}$ & $\begin{array}{l}\text { BG, BMM, BP, } \\
\text { BPQ, BQ, BTC, } \\
\text { BTP, BTSC, PAL, } \\
\text { PAZ, VS }\end{array}$ & $0-2360$ \\
\hline & Orthopappus angustifolius (Sw.) Gleason & $\begin{array}{l}\text { Chis., Gro., Oax., S.L.P., Ver., Centro a } \\
\text { Sudamérica, Antillas }\end{array}$ & BPQ, PAZ, VS & $25-1200$ \\
\hline & $\begin{array}{l}\text { Pseudelephantopus spicatus (Juss. ex Aubl.) } \\
\text { Rohr }\end{array}$ & $\begin{array}{l}\text { Camp., Chis., Col., Dgo., Gro., Hgo., Jal., } \\
\text { Méx., Mich., Mor., Nay., Oax., Pue., } \\
\text { Qro., S.L.P., Sin., Son., Tab., Ver., Yuc., } \\
\text { Estados Unidos de América, Centro } \\
\text { a Sudamérica, Antillas, África, Asia, } \\
\text { Australia }\end{array}$ & $\begin{array}{l}\text { BG, BJ, BMM, BP, } \\
\text { BPQ, BQ, BTC, } \\
\text { BTP, BTSC, PAZ, } \\
\text { VS }\end{array}$ & $0-2200$ \\
\hline \multirow[t]{11}{*}{ Leiboldiinae } & * Bolanosa coulteri A. Gray & Gto., Jal., Mich., Nay., Zac. & $\begin{array}{l}\text { BMM, BPQ, BQ, } \\
\text { BTC, BTSC, PAZ, } \\
\text { VS }\end{array}$ & $520-2139$ \\
\hline & $\begin{array}{l}\text { * Leiboldia guerreroana (S.B. Jones) H. } \\
\text { Rob. }\end{array}$ & Gro., Oax. & $\begin{array}{l}\text { BMM, BP, BPQ, } \\
\text { BQ, BTC }\end{array}$ & $800-1950$ \\
\hline & * Leiboldia serrata (D. Don) Gleason & Hgo., Oax., Pue., Qro., Ver. & $\begin{array}{l}\text { BMM, BP, BPQ, } \\
\text { BTP, BTSC, VS }\end{array}$ & $270-2200$ \\
\hline & $\begin{array}{l}\text { * Lepidonia alba Redonda-Mart. \& E. } \\
\text { Martínez }\end{array}$ & Chis. & BMM & 2162 \\
\hline & $\begin{array}{l}\text { * Lepidonia callilepis (Gleason) H. Rob. \& } \\
\text { V.A. Funk }\end{array}$ & Gro., Oax. & $\begin{array}{l}\mathrm{BG}, \mathrm{BMM}, \mathrm{BP} \\
\mathrm{BPQ}, \mathrm{BQ}\end{array}$ & $540-2400$ \\
\hline & $\begin{array}{l}\text { * Lepidonia jonesii (B.L. Turner) H. Rob. \& } \\
\text { V.A. Funk }\end{array}$ & Oax. & $\begin{array}{l}\text { BMM, BPQ, BTP, } \\
\text { VS }\end{array}$ & $1170-2360$ \\
\hline & $\begin{array}{l}\text { * Lepidonia mexicana (Less.) H. Rob. \& } \\
\text { V.A. Funk }\end{array}$ & Pue., Ver. & BG, BMM, BQ, BTP & $755-1700$ \\
\hline & $\begin{array}{l}\text { Lepidonia salvinae (Hemsl.) H. Rob. \& V.A. } \\
\text { Funk }\end{array}$ & Chis., Guatemala & BMM, BPQ, VS & $454-2600$ \\
\hline & $\begin{array}{l}\text { * Lepidonia wendtiana (B.L. Turner) } \\
\text { Redonda-Mart. \& Villaseñor }\end{array}$ & Oax. & BMM, ВТP & $600-1000$ \\
\hline & $\begin{array}{l}\text { * Stramentopappus congestiflorus Redonda- } \\
\text { Mart. \& Villaseñor }\end{array}$ & Oax. & $\mathrm{BMM}, \mathrm{BP}$ & $2300-2850$ \\
\hline & $\begin{array}{l}\text { * Stramentopappus pooleae (B.L. Turner) H. } \\
\text { Rob. \& V.A. Funk }\end{array}$ & Oax. & BMM, BPQ, BQ & $1090-2850$ \\
\hline Lepidaploinae & Harleya oxylepis (Benth.) S.F. Blake & Chis., Q. Roo, Tab., Yuc., Centroamérica & BTP, BTSC & $100-150$ \\
\hline
\end{tabular}


Cuadro 2: Continuación.

\begin{tabular}{|c|c|c|c|c|}
\hline Subtribu & Especie & Distribución por estado & Tipo de vegetación & Altitud \\
\hline & $\begin{array}{l}\text { * Lepidaploa boquerona (B.L. Turner) H. } \\
\text { Rob. }\end{array}$ & Chis. & BMM, BPQ & $2255-2600$ \\
\hline & Lepidaploa canescens (Kunth) H. Rob. & $\begin{array}{l}\text { Chis., Col., Gro., Hgo., Jal., Nay., Oax., } \\
\text { Qro., Q. Roo, S.L.P., Ver., Yuc., Centro a } \\
\text { Sudamérica, Antillas }\end{array}$ & $\begin{array}{l}\text { BG, BMM, BPQ, } \\
\text { BQ, BTC, BTSC, } \\
\text { PAZ, VS }\end{array}$ & $5-2200$ \\
\hline & $\begin{array}{l}\text { * Lepidaploa diazlunana (B.L. Turner) H. } \\
\text { Rob. }\end{array}$ & Gro. & BMM, BTP, BTSC & $885-1650$ \\
\hline & * Lepidaploa koelzii (McVaugh) H. Rob. & Col. & BTC & $400-450$ \\
\hline & Lepidaploa polypleura (S.F. Blake) H. Rob. & Chis., Oax., Centroamérica & $\begin{array}{l}\text { BMM, BPQ, BQ, } \\
\text { BTP }\end{array}$ & $700-2200$ \\
\hline & Lepidaploa remotiflora (Rich.) H. Rob. & Chis., Centro a Sudamérica, Antillas & BTP & $100-900$ \\
\hline & Lepidaploa salzmanii (DC.) H. Rob. & $\begin{array}{l}\text { Chis., Gro., Hgo., Oax., Pue., Tab., Ver., } \\
\text { Centro a Sudamérica }\end{array}$ & $\begin{array}{l}\text { BG, BMM, BP, BQ, } \\
\text { BTP, BTSC, VS }\end{array}$ & $0-1700$ \\
\hline & Lepidaploa tortuosa (L.) H. Rob. & $\begin{array}{l}\text { Camp., Chis., Gro., Hgo., Oax. Pue., Qro., } \\
\text { S.L.P., Tab., Ver., Centroamérica }\end{array}$ & $\begin{array}{l}\text { BG, BMM, BP. } \\
\text { BPQ, BQ, BTC, } \\
\text { BTP, BTSC, MAN, } \\
\text { MX, PAZ, VS }\end{array}$ & $0-1870$ \\
\hline & Lepidaploa uniflora (Mill.) H. Rob. & Camp., Q. Roo, Tab., Yuc., Centroamérica & BTC, BTSC, PAZ & $12-280$ \\
\hline & Stenocephalum jucundum (Gleason) H. Rob. & Chis., Oax., Centro a Sudamérica & $\begin{array}{l}\text { BG, BP, BPQ, BQ, } \\
\text { BTC, BTSC, PAZ }\end{array}$ & $150-1100$ \\
\hline & + Struchium sparganophorum (L.) Kuntze & $\begin{array}{l}\text { Chis., Tab. Centro a Sudamérica, Antillas, } \\
\text { África, Asia, Australia }\end{array}$ & BTP, BTSC & $0-400$ \\
\hline \multirow[t]{13}{*}{ Piptocaphinae } & $\begin{array}{l}\text { * Critoniopsis autumnalis (McVaugh) H. } \\
\text { Rob. }\end{array}$ & Jal. & $\begin{array}{l}\text { BPQ, BQ, BTC, } \\
\text { BTSC }\end{array}$ & $500-1000$ \\
\hline & * Critoniopsis baadii (McVaugh) H. Rob. & Jal. & BMM, BPQ, BTC & $950-2000$ \\
\hline & $\begin{array}{l}\text { * Critoniopsis barbinervis (Sch. Bip.) H. } \\
\text { Rob. }\end{array}$ & Dgo., Sin. & $\mathrm{BP}, \mathrm{BPQ}, \mathrm{BQ}, \mathrm{BTC}$ & $100-2400$ \\
\hline & * Critoniopsis feddemae (McVaugh) & Nay. & BTC & 1000 \\
\hline & $\begin{array}{l}\text { Villaseñor \& Redonda-Mart. } \\
\text { * Critoniopsis foliosa (Benth.) H. Rob. }\end{array}$ & $\begin{array}{l}\text { Ags., Chih., Dgo., Gto., Jal., Nay., S.L.P., } \\
\text { Zac. }\end{array}$ & $\begin{array}{l}\text { BG, BP, BPQ, BQ, } \\
\text { BTC, MX, PAZ }\end{array}$ & $800-2500$ \\
\hline & $\begin{array}{l}\text { * Critoniopsis heydeana (J.M. Coult.) H. } \\
\text { Rob. }\end{array}$ & Chis., Oax., Qro., S.L.P., Ver. & $\begin{array}{l}\text { BG, BMM, BPQ, } \\
\text { BQ, BTC, BTSC }\end{array}$ & $850-2052$ \\
\hline & Critoniopsis leiocarpa (DC.) H. Rob. & $\begin{array}{l}\text { Chis., Gro., Mich., Oax., Ver., } \\
\text { Centroamérica }\end{array}$ & $\begin{array}{l}\text { BMM, BP, BPQ, } \\
\text { BQ, BTC, BTP, VS }\end{array}$ & $400-2500$ \\
\hline & * Critoniopsis littoralis (Brandegee) H. Rob. & Col. & BTC & $550-800$ \\
\hline & $\begin{array}{l}\text { * Critoniopsis macphersonii (S.B. Jones \& } \\
\text { Stutts) H. Rob. }\end{array}$ & Jal. & BMM, BPQ & $850-1150$ \\
\hline & $\begin{array}{l}\text { * Critoniopsis macvaughii (S.B. Jones) H. } \\
\text { Rob. }\end{array}$ & Gro., Oax., Pue. & BPQ, BQ, BTC, MX & $1500-2500$ \\
\hline & * Critoniopsis obtusa (Gleason) H. Rob. & Hgo., N.L., Qro., S.L.P., Tam., Ver. & $\begin{array}{l}\text { BG, BMM, BP, } \\
\text { BPQ, BQ, MX, VS }\end{array}$ & $10-2400$ \\
\hline & * Critoniopsis oolepis (S.F. Blake) H. Rob. & Camp., Q. Roo, Yuc. & $\begin{array}{l}\text { BTC, BTP, BTSC, } \\
\text { VS }\end{array}$ & $5-74$ \\
\hline & * Critoniopsis ovata (Gleason) H. Rob. & Chih., Dgo. & BTC, MX & $600-1545$ \\
\hline
\end{tabular}


Cuadro 2: Continuación.

\begin{tabular}{|c|c|c|c|c|}
\hline Subtribu & Especie & Distribución por estado & Tipo de vegetación & Altitud \\
\hline & * Critoniopsis pallens (Sch. Bip.) H. Rob. & Gro., Méx., Mich., Mor. & BTC, MX & $800-1200$ \\
\hline & * Critoniopsis pugana (S.B. Jones \& Stutts) & Jal. & BMM & $1500-2000$ \\
\hline & $\begin{array}{l}\text { H. Rob. } \\
\text { * Critoniopsis salicifolia (DC.) H. Rob. }\end{array}$ & $\begin{array}{l}\text { Col., Cd. Mx., Gro., Jal., Méx., Mich., } \\
\text { Mor., Nay., Oax., Pue. }\end{array}$ & $\begin{array}{l}\text { BG, BMM, BP, } \\
\text { BPQ, BQ, BTC, } \\
\text { BTSC, MX }\end{array}$ & $120-2450$ \\
\hline & Critoniopsis shannonii (J.M. Coult.) H. Rob. & Chis., Guatemala & BMM & $2200-2500$ \\
\hline & $\begin{array}{l}\text { * Critoniopsis solorzanoana (Rzed. \& } \\
\text { Calderón) H. Rob. }\end{array}$ & Mich. & BMM & 2300 \\
\hline & $\begin{array}{l}\text { * Critoniopsis tarchonanthifolia (DC.) H. } \\
\text { Rob. }\end{array}$ & Oax. & $\mathrm{BPQ}, \mathrm{BQ}$ & $2250-2600$ \\
\hline & $\begin{array}{l}\text { * Critoniopsis tomentosa (La Llave \& Lex.) } \\
\text { H. Rob. }\end{array}$ & $\begin{array}{l}\text { Gto., Gro., Jal., Méx., Mich., Oax., Qro., } \\
\text { Ver., Zac. }\end{array}$ & $\begin{array}{l}\text { BMM, BP, BPQ, } \\
\text { BQ, BTC, MX, PAZ, } \\
\text { VS }\end{array}$ & $1200-2500$ \\
\hline & Critoniopsis triflosculosa (Kunth) H. Rob. & $\begin{array}{l}\text { Chis., Col., Dgo., Gro., Jal., Mich., } \\
\text { Mor., Nay., Oax., Sin., Son., Ver., } \\
\text { Centroamérica }\end{array}$ & $\begin{array}{l}\text { BG, BMM, BPQ, } \\
\text { BQ, BTC, BTSC, } \\
\text { MX, PAL, PAZ, VS }\end{array}$ & $10-1800$ \\
\hline & * Critoniopsis uniflora (Sch. Bip.) H. Rob. & $\begin{array}{l}\text { Ags., Col., Dgo., Gro., Jal., Méx., Nay., } \\
\text { Oax., Pue., Ver., Zac. }\end{array}$ & $\begin{array}{l}\text { BG, BMM, BP, } \\
\text { BPQ, BQ, BTC, } \\
\text { MX, PAL, PAZ, VS }\end{array}$ & $900-2400$ \\
\hline & $\begin{array}{l}\text { * Critoniopsis villaregalis (Carvajal) H. } \\
\text { Rob. }\end{array}$ & Jal., Mich., Zac. & $\begin{array}{l}\text { BPQ, BQ, BTC, } \\
\text { MX, PAZ, VS }\end{array}$ & $850-2000$ \\
\hline & Piptocarpha poeppigiana (DC.) Baker & Chis., Oax., Ver., Centro a Sudamérica & $\begin{array}{l}\text { BMM, BTP, BTSC, } \\
\text { VS }\end{array}$ & $50-1200$ \\
\hline Rolandrinae & Spiracantha cornifolia Kunth & $\begin{array}{l}\text { Camp., Q. Roo., Tab., Ver., Yuc., Centro a } \\
\text { Sudamérica, Antillas }\end{array}$ & $\begin{array}{l}\text { BTC, BTP, BTSC, } \\
\text { VS }\end{array}$ & $10-300$ \\
\hline \multirow[t]{8}{*}{ Vernoniinae } & Cyrtocymura scorpioides (Lam.) H. Rob. & $\begin{array}{l}\text { Camp., Q. Roo, Yuc., Centro a } \\
\text { Sudamérica }\end{array}$ & BTP, BTSC & $30-50$ \\
\hline & Vernonanthura cordata (Kunth) H. Rob. & $\begin{array}{l}\text { Col., Gro., Jal., Méx., Mich., Mor., Nay., } \\
\text { Oax., Ver. }\end{array}$ & $\begin{array}{l}\text { BG, BMM, BP, } \\
\text { BPQ, BQ, BTC, } \\
\text { BTSC, MX, PAZ, } \\
\text { VS }\end{array}$ & $450-2800$ \\
\hline & Vernonanthura patens (Kunth) H. Rob. & $\begin{array}{l}\text { Camp., Chis., Col., Gro., Hgo., Jal., Mor., } \\
\text { Nay., Oax., Pue., Qro., S.L.P., Sin., Ver., } \\
\text { Tab., Yuc., Centro a Sudamérica. }\end{array}$ & $\begin{array}{l}\text { BG, BMM, BP, } \\
\text { BPQ, BQ, BTC, } \\
\text { BTP, BTSC, PAZ, } \\
\text { SAB, VS }\end{array}$ & $5-2200$ \\
\hline & * Vernonia alamanii DC. & $\begin{array}{l}\text { Gto., Gro., Hgo., Jal., Méx., Mich., Mor., } \\
\text { Pue., Qro., S.L.P., Ver. }\end{array}$ & $\begin{array}{l}\text { BA,BG, BJ, BMM, } \\
\text { BP, BPQ, BQ, BTC, } \\
\text { MX, PAZ, VS }\end{array}$ & $1050-2600$ \\
\hline & * Vernonia barclayi H. Rob. \& C.F. Reed & Son. & BQ & 1800 \\
\hline & *Vernonia bealliae McVaugh & Jal., Mich. & $\begin{array}{l}\text { BA, BG, BMM, BP, } \\
\text { BPQ, BQ, BTC, VS }\end{array}$ & $1100-2600$ \\
\hline & *Vernonia boolleana Sch. Bip. & Dgo., Nay., Sin. & $\mathrm{BPQ}, \mathrm{BQ}$ & 1700 \\
\hline & $\begin{array}{l}\text { * Vernonia confusa } \text { Redonda-Mart., } \\
\text { Villaseñor \& A. Campos }\end{array}$ & Oax. & BMM, BP, BPQ & $1260-1790$ \\
\hline
\end{tabular}


Cuadro 2: Continuación.

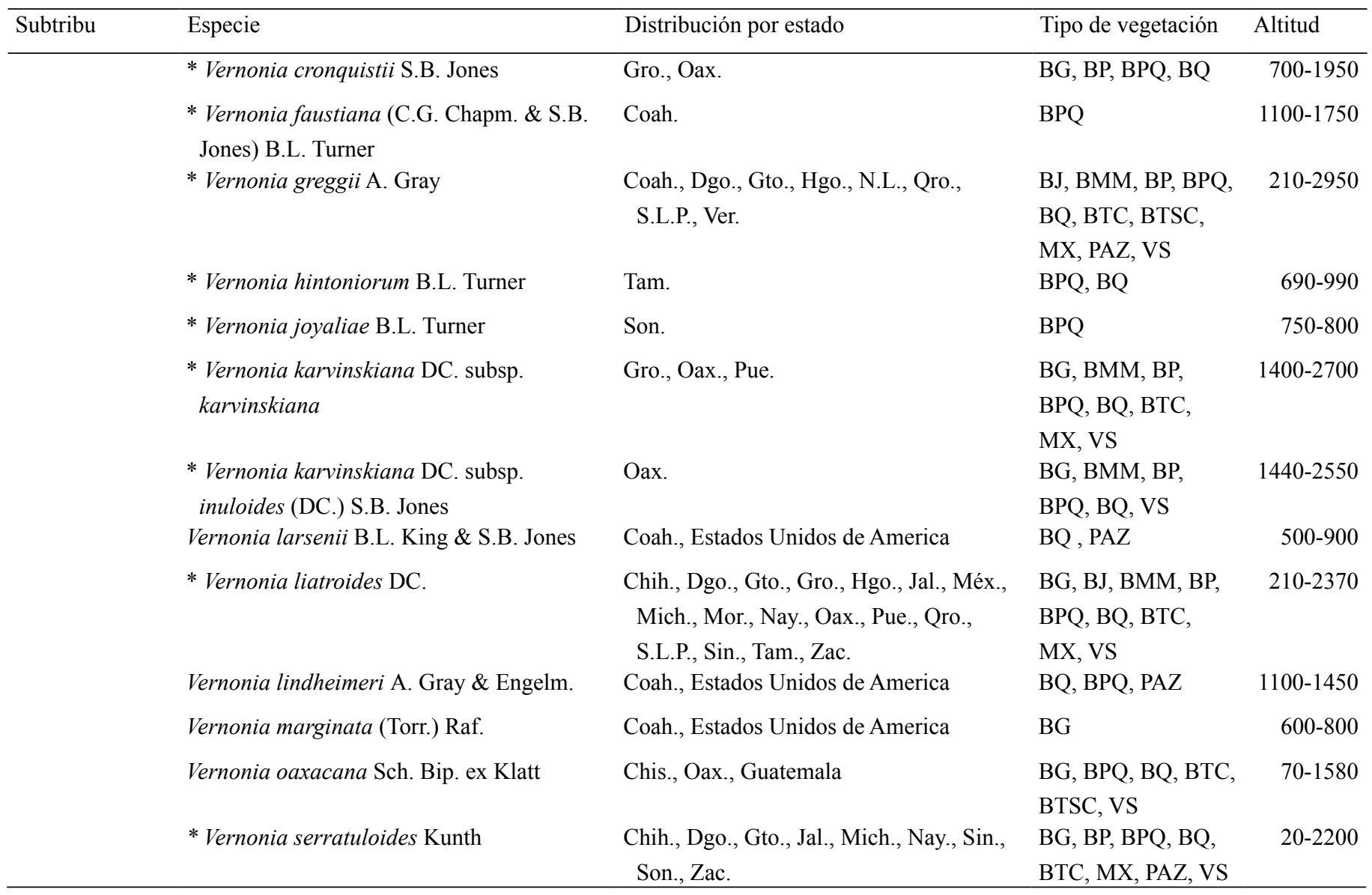

Cuadro 3: Formas de vida y fenología de las especies de Vernonieae presentes en México. Las especies endémicas están precedidas por un asterisco y las introducidas por el signo + .

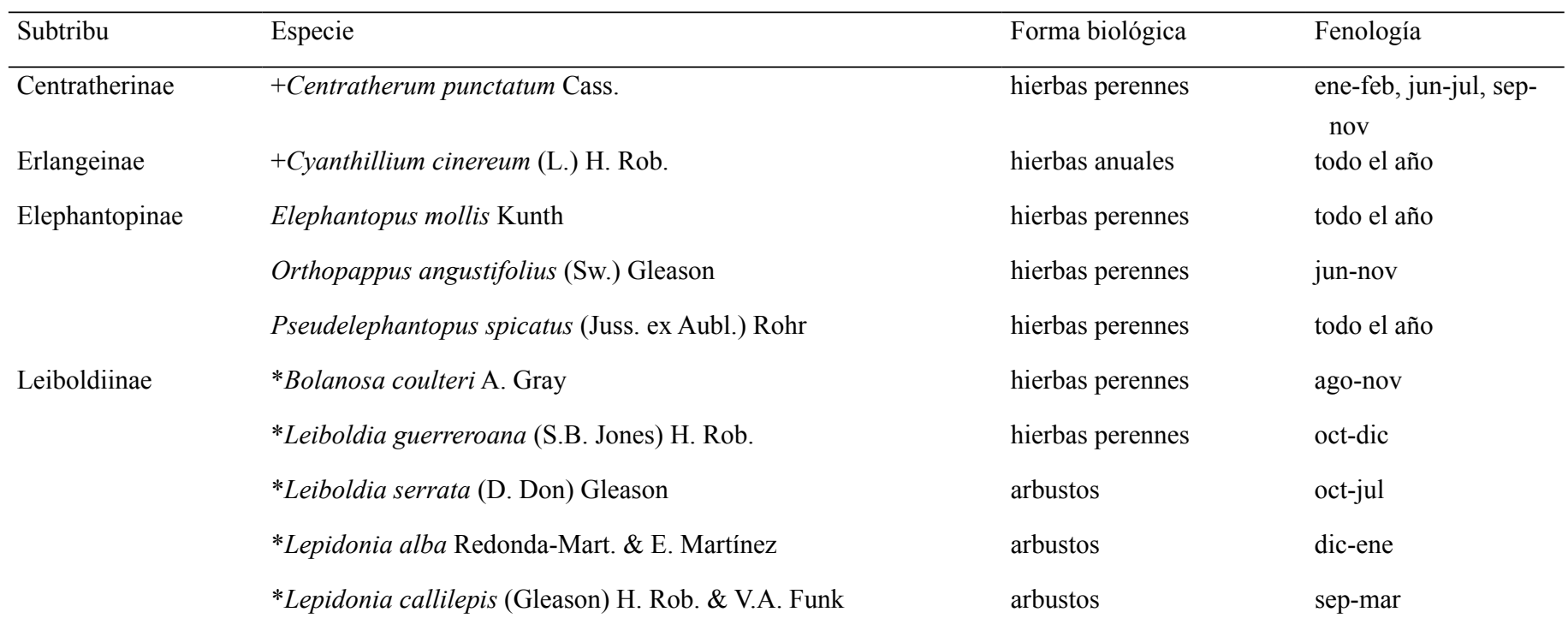


Cuadro 3: Continuación.

\begin{tabular}{|c|c|c|c|}
\hline Subtribu & Especie & Forma biológica & Fenología \\
\hline & *Lepidonia jonesii (B.L. Turner) H. Rob. \& V.A. Funk & arbustos & nov-may \\
\hline & *Lepidonia mexicana (Less.) H. Rob. \& V.A. Funk & arbustos & mar-may, jul-sep \\
\hline & Lepidonia salvinae (Hemsl.) H. Rob. \& V.A. Funk & arbustos & jul-mar \\
\hline & *Lepidonia wendtiana (B.L. Turner) Redonda-Mart. \& Villaseñor & árboles & abr \\
\hline & *Stramentopappus congestiflorus Redonda-Mart. \& Villaseñor & arbustos & may-sep \\
\hline & *Stramentopappus pooleae (B.L. Turner) H. Rob. \& V.A. Funk & arbustos & ene-abr, jul-ago \\
\hline \multirow[t]{13}{*}{ Lepidaploinae } & Harleya oxylepis (Benth.) S.F. Blake & hierbas perennes & feb-jun \\
\hline & *Lepidaploa boquerona (B.L. Turner) H. Rob. & arbustos escandentes & nov-dic \\
\hline & Lepidaploa canescens (Kunth) H. Rob. & arbustos escandentes & nov-ago \\
\hline & *Lepidaploa diazlunana (B.L. Turner) H. Rob. & arbustos erectos o & dic-may \\
\hline & & escandentes & \\
\hline & *Lepidaploa koelzii (McVaugh) H. Rob. & arbustos erectos & oct-dic \\
\hline & Lepidaploa polypleura (S.F. Blake) H. Rob. & árboles & oct-abr \\
\hline & Lepidaploa remotiflora (Rich.) H. Rob. & hierbas perennes & ene-abr \\
\hline & Lepidaploa salzmanii (DC.) H. Rob. & arbustos erectos & nov-may \\
\hline & Lepidaploa tortuosa (L.) H. Rob. & arbustos escandentes & todo el año \\
\hline & Lepidaploa uniflora (Mill.) H. Rob. & arbustos erectos & nov-may \\
\hline & Stenocephalum jucundum (Gleason) H. Rob. & hierbas perennes & jul-dic \\
\hline & + Struchium sparganophorum (L.) Kuntze & hierbas perennes & feb-jun \\
\hline \multirow[t]{14}{*}{ Piptocaphinae } & *Critoniopsis autumnalis (McVaugh) H. Rob. & hierbas perennes & oct-dic \\
\hline & *Critoniopsis baadii (McVaugh) H. Rob. & arbustos & feb-abr, dic \\
\hline & *Critoniopsis barbinervis (Sch. Bip.) H. Rob. & arbustos & oct-mar \\
\hline & *Critoniopsis feddemae (McVaugh) Villaseñor \& Redonda-Mart. & hierbas perennes & ago \\
\hline & *Critoniopsis foliosa (Benth.) H. Rob. & arbustos & todo el año \\
\hline & *Critoniopsis heydeana (J.M. Coult.) H. Rob. & arbustos & ene-abr \\
\hline & Critoniopsis leiocarpa (DC.) H. Rob. & arbustos & oct-jun \\
\hline & *Critoniopsis littoralis (Brandegee) H. Rob. & arbustos escandentes & mar-jun \\
\hline & *Critoniopsis macphersonii (S.B. Jones \& Stutts) H. Rob. & arbustos & feb-mar \\
\hline & *Critoniopsis macvaughii (S.B. Jones) H. Rob. & arbustos & sep-mar \\
\hline & *Critoniopsis obtusa (Gleason) H. Rob. & arbustos & jul-ene \\
\hline & *Critoniopsis oolepis (S.F. Blake) H. Rob. & arbustos & dic-abr \\
\hline & *Critoniopsis ovata (Gleason) H. Rob. & arbustos & mar-abr \\
\hline & *Critoniopsis pallens (Sch. Bip.) H. Rob. & arbustos & ene-mar \\
\hline
\end{tabular}


Cuadro 3: Continuación.

\begin{tabular}{|c|c|c|c|}
\hline Subtribu & Especie & Forma biológica & Fenología \\
\hline & *Critoniopsis salicifolia (DC.) H. Rob. & arbustos & todo el año \\
\hline & Critoniopsis shannonii (J.M. Coult.) H. Rob. & arbustos & dic-feb \\
\hline & *Critoniopsis solorzanoana (Rzed. \& Calderón) H. Rob. & árboles & mar \\
\hline & ${ }^{*}$ Critoniopsis tomentosa (La Llave \& Lex.) H. Rob. & arbustos & sep-abr \\
\hline & Critoniopsis triflosculosa (Kunth) H. Rob. & arbustos & sep-abr \\
\hline & *Critoniopsis uniflora (Sch. Bip.) H. Rob. & arbustos & oct-abr \\
\hline \multirow[t]{16}{*}{ Vernoniinae } & Cyrtocymura scorpioides (Lam.) H. Rob. & arbustos escandentes & feb-may \\
\hline & Vernonanthura cordata (Kunth) H. Rob. & árboles & dic-may \\
\hline & Vernonanthura patens (Kunth) H. Rob. & árboles & todo el año \\
\hline & *Vernonia alamanii DC. & arbustos & sep-jun \\
\hline & *Vernonia barclayi H. Rob. \& C.F. Reed & arbustos & abr-may \\
\hline & *Vernonia bealliae McVaugh & arbustos & nov-may \\
\hline & *Vernonia hintoniorum B.L. Turner & arbustos & ago-nov \\
\hline & *Vernonia joyaliae B.L. Turner & hierbas perennes & abr-may \\
\hline & *Vernonia karvinskiana DC. subsp. karvinskiana & arbustos & jul-ene \\
\hline & *Vernonia karvinskiana DC. subsp. inuloides (DC.) S.B. Jones & arbustos & sep-may \\
\hline & Vernonia larsenii B.L. King \& S.B. Jones & hierbas perennes & jul-oct \\
\hline & *Vernonia liatroides DC. & hierbas perennes & ago-abr \\
\hline & Vernonia lindheimeri A. Gray \& Engelm. & hierbas perennes & jun-ago \\
\hline & Vernonia marginata (Torr.) Raf. & hierbas perennes & jul-ago \\
\hline & Vernonia oaxacana Sch. Bip. ex Klatt & arbustos & nov-jun \\
\hline & *Vernonia serratuloides Kunth & hierbas perennes & ago-dic \\
\hline
\end{tabular}




\section{DISCUSIÓN Y CONCLUSIONES}

A pesar de que en México la tribu Vernonieae no es un grupo tan diverso como ocurre con otras tribus de Asteraceae, entre las que destacan Heliantheae s.l. (117 géneros/1021 especies), Eupatorieae (32 géneros/541 especies), Astereae (43 géneros/400 especies), Helenieae s.1. (58 géneros/239 especies) y Senecioneae (21 géneros/240 especies) (Villarreal y Villaseñor, 2004), es importante señalar que más de $60 \%$ de las especies de Vernonieae presentes en el país son endémicas y únicamente 4.2\% corresponde a taxones introducidos. Cuatro especies, Critoniopsis feddemae, Lepidonia alba, Vernonia barclayi y $V$. joyalieae, se conocen únicamente de la colección tipo, mientras que tres: Lepidaploa boquerona, Lepidonia wendtiana y Vernonia confusa se conocen de la colección tipo y una o dos colectas adicionales. Esto refleja el nivel de rareza de varios taxones de la tribu, que en el caso particular de las especies antes mencionadas correspondería al tipo 4, según la propuesta de Rabinowitz et al. (1986), pues tienen distribución geográfica restringida, alta especificidad del hábitat y las poblaciones son pequeñas (no más de 5 individuos, obs. pers.). Este nivel de rareza también es común en especies que se conocen de varias colectas pero restringen su distribución a unas cuantas localidades de un solo estado, como ocurre con la mayor parte de las especies de la subtribu Leiboldiinae (Redonda-Martínez, 2016).

Los géneros actualmente reconocidos en Vernonieae corresponden en su mayoría a géneros segregados a partir de secciones de Vernonia s.l. La mayoría son fáciles de reconocer e incluso los caracteres que los distinguen son diagnósticos; por ejemplo Lepidaploa se diferencia de los géneros Vernonanthura y Cyrtocymura, que también presentan inflorescencias escorpioideas, por las brácteas asociadas a las cabezuelas (Redonda-Martínez y Villaseñor, 2011). El género Stramentopappus se distingue de Leiboldia y Lepidonia, por el vilano pajizo multiseriado y las cipselas obovadas (Redonda-Martínez y Villaseñor, 2014; Redonda-Martínez, 2016). Lepidonia se caracteriza por los filarios con el ápice ampliamente ovado-apiculado (Robinson y Funk, 1987; Redonda-Martínez, 2016) y Lei- boldia por la presencia de glándulas en la base de la cipsela por arriba del carpopodio (Redonda-Martínez, 2016, Redonda-Martínez et al., 2016a). El género Critoniopsis también es fácil de reconocer por los filarios deciduos en las cabezuelas maduras.

La mayor controversia en los géneros segregados de Vernonia s.1., se presenta en Vernonanthura, el cual según Robinson (1992) se diferencia de Vernonia s.s. por las inflorescencias piramidales y la presencia de anteras caudadas. Sin embargo, este último carácter no se presenta en las especies mexicanas transferidas a dicho género, pues las anteras caudadas tal y como las definió el mismo Robinson (1983) son aquellas que presentan una prolongación por debajo del conectivo constituida exclusivamente por células estériles. Si bien la base de las anteras de las vernonanthuras mexicanas presenta proyecciones basales, éstas no son estériles porque tienen granos de polen, por lo cual son anteras calcaradas más no caudadas (Robinson, 1983, Roque et al., 2009). Los caracteres que ayudan a definir de una mejor manera los límites entre Vernonia y Vernonanthura son el hábito y el tipo de inflorescencia, las especies mexicanas de Vernonanthura s.s. son arborescentes o árboreas, con inflorescencias cimoso escorpioideas, mientras que Vernonia presenta formas de vida herbáceas perennes o arbustivas con inflorescencias corimbiformes, umbeliformes, cimosas o tirsoides (Cuadro 4). Esta es la razón por la cual en este trabajo se reconocen únicamente dos especies de Vernonanthura y las cinco restantes se transfieren nuevamente a Vernonia, como se ha propuesto en trabajos previos (Redonda-Martínez, 2006; RedondaMartínez y Villaseñor, 2009).

Tres taxones que Robinson (1999) considera válidos (Vernonanthura deppeana (Less.) H. Rob. $\equiv$ Vernonanthura patens (Kunth) H. Rob., Vernonanthura sinclairii (Benth.) H. Rob. $\equiv$ Vernonanthura serratuloides (Kunth) H. Rob., Critoniopsis duncanii (S.B. Jones) H. Rob. $\equiv$ Critoniopsis foliosa (Benth.) H. Rob.), se reducen a sinonimia debido a la similitud morfológica que presentan con los ejemplares tipo de las especies reconocidas como válidas en este trabajo. Además 
Cuadro 4: Diferencias morfológicas entre las especies mexicanas de Vernonanthura H. Rob y Vernonia Schreb.

\begin{tabular}{|c|c|c|c|c|}
\hline Especie & Forma de vida & Tipo de inflorescencia & Color flores & Indumento lóbulos corola \\
\hline Vernonanthura cordata (Kunth) H. Rob. & árboles & cima escorpioidea & blanco & glandular \\
\hline Vernonanthura patens (Kunth) H. Rob. & árboles & cima escorpioidea & blanco & ausente \\
\hline *Vernonia alamanii DC. & arbustos & corimbiforme, umbeliforme & morado & glandular \\
\hline *Vernonia barclayi H. Rob. \& C.F. Reed & arbustos & corimbiforme & morado & glandular \\
\hline *Vernonia bealliae McVaugh & arbustos & corimbiforme & morado & glandular \\
\hline *Vernonia boolleana Sch. Bip. & hierbas perennes & corimbiforme & morado & glandular \\
\hline $\begin{array}{l}\text { *Vernonia confusa Redonda-Mart., Villaseñor \& A. } \\
\text { Campos }\end{array}$ & hierbas perennes & corimbiforme & morado & glandular \\
\hline *Vernonia cronquistii S.B. Jones & hierbas perennes & corimbiforme & morado & ausente \\
\hline $\begin{array}{l}\text { *Vernonia faustiana (C.G. Chapm. \& S.B. Jones) B.L. } \\
\text { Turner }\end{array}$ & hierbas perennes & corimbiforme & morado & glandular \\
\hline *Vernonia greggii A. Gray & hierbas perennes & cimosa & morado & glandular \\
\hline *Vernonia hintoniorum B.L. Turner & arbustos & corimbiforme & morado & glandular \\
\hline *Vernonia joyaliae B.L. Turner & & corimbiforme & morado & glandular \\
\hline *Vernonia karvinskiana DC. subsp. karvinskiana & arbustos & corimbiforme & morado & glandular \\
\hline $\begin{array}{l}\text { *Vernonia karvinskiana DC. subsp. inuloides (DC.) S.B. } \\
\text { Jones }\end{array}$ & arbustos & corimbiforme & morado & glandular \\
\hline Vernonia larsenii B.L. King \& S.B. Jones & hierbas perennes & corimbiforme & morado & glandular \\
\hline *Vernonia liatroides DC. & hierbas perennes & tirsoide & morado & glandular \\
\hline Vernonia lindheimeri A. Gray \& Engelm. & hierbas perennes & corimbiforme & morado & glandular \\
\hline Vernonia marginata (Torr.) Raf. & hierbas perennes & corimbiforme & morado & glandular \\
\hline Vernonia oaxacana Sch. Bip. ex Klatt & arbustos & corimbiforme & morado & glandular \\
\hline *Vernonia serratuloides Kunth & hierbas perennes & tirsoide & morado & glandular \\
\hline
\end{tabular}

también tienen una diversidad de tricomas similar a la de las especies válidas y como se ha propuesto en otros trabajos, esta característica ha sido importante para reconocer y delimitar taxones en Vernonieae (Hunter y Austin, 1967; Jones, 1973; Faust y Jones, 1973; King y Jones, 1975; Robinson, 1977; Narayana, 1979; Redonda-Martínez y Villaseñor, 2009; Redonda-Martínez et al., 2012, 2016). Aunque el nombre Lepidaploa arborescens (L.) H. Rob. es válido, se excluye de este trabajo porque es un nombre restringido a las Antillas y los taxones colectados en la región continental que se han identificaco bajo ese nombre corresponden a $L$. canescens (Keeley, 1982).

\section{CONTRIBUCIÓN DE AUTOR}

RRM concibió, diseñó y realizó el estudio, así como la escritura, revisión, corrección y aprobación del manuscrito.

\section{FINANCIAMIENTO}

Este estudio fue apoyado por el Consejo Nacional de Ciencia y Tecnología (CONACYT) con la beca (263523) otorgada para realizar estudios de posgrado, y el Posgrado en Ciencias Biológicas de la Universidad Nacional Autónoma de México, mediante el Programa de Apoyo a los Estudios de Posgrado (PAEP 2014). 


\section{AgradeCIMIENTOS}

José Luis Villaseñor, Teresa Terrazas, Rosaura Grether y Martín García-Varela revisaron una versión preliminar del manuscrito y aportaron valiosas sugerencias para mejorarlo. Georgina Ortega-Leite apoyó en la búsqueda de algunas referencias bibliográficas. Berenit MendozaGarfías encargada del microscopio electrónico de barrido del Instituto de Biología (UNAM), colaboró en la obtención de material fotográfico. Esteban Martínez-Salas (IBUNAM) y Jesús López-Santiago (UNSIJ) proporcionaron fotografías de algunas especies ilustradas. María del Rosario García Peña facilitó la consulta del material tipo depositado en la colección del Herbario Nacional de México (MEXU) y gestionó los préstamos de algunos herbarios extranjeros. Finalmente, se agradece a los curadores de los herbarios CIB (Hermann Bojórquez), CIIDIR (Socorro González Elizondo), CTES (Massimiliano Dematteis), ENCB (María de la Luz Arreguín Sánchez), F (Christine J. Niezgoda), FCME (Jaime Jiménez Ramírez), IEB (Sergio Zamudio Ruíz), MEXU (David Gernandt), MO (James C. Solomon), TEX (Thomas Wendt), US (Rusty Russell), XAL (Gonzalo Castillo Campos) y XALU (Mario Vázquez) por las facilidades otorgadas para consultar las colecciones y aprobar los préstamos correspondientes.

\section{LITERATURA CITADA}

Bentham, G. 1873. Ordo LXXXVIII: Compositae. In: Bentham, G. y J. D. Hooker (eds.). Genera Plantarum. Reeve and Co. London, UK. 2(1): 163-533.

Bremer, K. 1987. Tribal interrelationships of the Asteraceae. Cladistics 3: 210-253.

Cassini, A. H. G. 1819. Suite de Sixième mémorie sur la famille des Synanthérées, contenant les caractères des tribus. Journal de Physique, de Chimie, d'Historie Naturelle et des Arts 88: 189-204.

Chapman, G. C. y S. B. Jones. 1978. Biosystematics of the Texanae Vernonias (Vernonieae: Compositae). Sida 7: 264281.

Faust, W. Z. y S. B. Jones. 1973. The systematic value of trichome complements in a North American group of Vernonia (Compositae). Rhodora 75: 517-528.
Funk, V. A., J. B. Randall, S. C. Keeley, R. Chan, L. Watson, B. Gemeinholzer, E. Schilling, J. L. Panero, B. G. Baldwin, N. García-Jacas, A. Sussana y R. K. Jansen. 2005. Everywhere but Antarctica: Using a supertree to understand the diversity and distribution of the Compositae. Biologiske Skrifter 55: 343-373.

Funk, V. A., A. A. Anderberg, B. G. Baldwin, R. J. Bayer, J. M. Bonifacio, I. Bretitwieser, L. Brouillet, R. Carbajal, R. Chan, A. X. P. Coutinho, D. J. Crawford, J. V. Crisci, M. O. Dillon, S. E. Freire, M. Galbany-Casals, N. García-Jacas, B. Gemeinholzer, M. Gruenstaeudl, H. V. Hansen, S. Himmelreich, J. W. Kadereit, M. Källersjö, V. Karaman-Castro, P. O. Karis, L. Katinas, S. C. Keeley, N. Kilian, R. T. Kimball, T. K. Lowrey, J. Lundberg, R. J. McKenzie, M. Tadesse, M. E. Mort, B. Nordenstam, C. Oberprieler, S. Ortiz, P. B. Pelser, C. P. Randle, H. Robinson, N. Roque, G. Sancho, J. C. Semple, M. Serrano, T. F. Stuessy, A. Sussana, M. Unwin, L. Urbatsch, E. Urtbey, J. Vallès, R. Vogt, S. Wagstaff, J. Ward y L. E. Watson. 2009. Compositae metatrees: the next generation. In: Funk, V. A., A. Sussana, T. F. Stuessy y R. J. Bayer (eds.). Systematics, Evolution and Biogeography of the Compositae. International Association For Plant Taxonomy (IAPT). Viena, Austria. Pp. 747-777.

Gleason, H. A. 1906. A revision of the North American Vernonieae. Bulletin of the New York Botanical Garden 4: 144-243.

Gleason, H. A. 1922. Vernonieae. North American Flora 33: 47110.

Gleason, H. A. 1923. Evolution and geographical distribution of the genus Vernonia in North America. American Journal of Botany 10(4): 187-202.

Hunter, G. E. y D. E. Austin. 1967. Evidence from trichome morphology of interspecific hybridization in Vernonia: Compositae. Brittonia 19(1): 38-41. DOI: http://dx.doi. org/10.2307/2805081

Jansen, R. K., H. J. Michaels y J. D. Palmer. 1991. Phylogeny and character evolution in the Asteraceae based on chloroplast DNA restriction site mapping. Systematic Botany 16(1): 98-115. DOI: http://dx.doi. org/10.2307/2418976 
Jones, S. B. 1973. Revision of Vernonia section Eremosis (Compositae) in North America. Brittonia 25(2): 86-115. DOI: http://dx.doi.org/10.2307/2805929

Jones, S. B. 1976. Revision of Vernonia (Compositae), subsection Paniculatae, series Umbelliformes of the Mexican highlands. Rhodora 78: 180-206.

Jones, S. B. 1977. Vernonieae systematic review. In: Heywood, V. H., J. B. Harborne y B. L. Turner (eds.). The Biology and Chemistry of the Compositae. Academic Press. London, UK. 1: 503-521.

Jones, S. B. 1979a. Synopsis and pollen morphology of Vernonia (Compositae: Vernonieae) in the New World. Rhodora 81: 425-447.

Jones, S. B. 1979b. Taxonomic revision of Vernonia section Leiboldia (Compositae: Vernonieae). Castanea 44(4): 229-237.

Jones, S. B. 1981. Synoptic classification and pollen morphology of Vernonia (Compositae: Vernonieae) in the Old World. Rhodora 83: 59-75.

Jones, S. B. y J. G. Stutts. 1981. Three new species of Vernonia (Compositae: Vernonieae) from Mexico. Brittonia 33(4): 544-546. DOI: http://dx.doi.org/10.2307/2806761

Keeley, S. C. 1982. Morphological Variation and Species Recognition in the Neotropical taxon Vernonia arborescens (Compositae). Systematic Botany 7: 71-84. DOI: http:// dx.doi.org/10.2307/2418654

Keeley, S. C. y B. L. Turner. 1990. A preliminary cladistic analysis of the genus Vernonia (Vernonieae: Asteraceae). In: Mabry, T. J. y G. Wagenitz (eds.). Research advances in the Compositae. Plant Systematics and Evolution Supplementum 4: 45-66. DOI: http://dx.doi.org/10.1007/978-37091-6928-5_3

Keeley, S. C. y R. K. Jansen, 1994. Chloroplast DNA restriction site variation in the Vernonieae (Asteraceae), an initial appraisal of the relationship of New and Old World taxa and the monophyly of Vernonia. Plant Systematics and Evolution 193: 249-265. DOI: http://dx.doi.org/10.1007/ BF00983554

Keeley, S. C. y H. Robinson. 2009. Vernonieae. In: Funk, V. A., A. Sussana, T. F. Stuessy y R. J. Bayer (eds.). Systematics, Evolution and Biogeography of the Compositae.
International Association For Plant Taxonomy (IAPT). Viena, Austria. Pp. 439-469.

Keeley, S. C., Z. H. Forsman y R. Chan. 2007. A phylogeny of the "evil tribe" (Vernonieae: Compositae) reveals Old/New World long distance dispersal: support from separate and combined congruent datasets (trnL-F, ndhF, ITS). Molecular Phylogenetics and Evolution 44(1): 89-103. DOI: http://dx.doi.org/10.1016/j. ympev.2006.12.024

Kim, K.-J. y R. K. Jansen. 1995. ndhF sequence evolution and the major clades in the sunflower family. Proceedings of the National Academy of Sciences of the United States of America 92(22): 10379-10383.

King, B. L. y S. B. Jones. 1975. The Vernonia lindheimeri complex (Compositae). Brittonia. 24: 74-86. DOI: http://dx. doi.org/10.2307/2805649

McVaugh, R. 1984. Vernonia. In: Anderson, W. R. (ed.). Flora Novo-Galiciana. The University of Michigan Press. Ann Arbor, USA. 12: 1013-1039.

Narayana, B. M. 1979. Taxonomic value of trichomes in Vernonia Schreb. (Asteraceae). Proceedings of the Indian Academy of Sciences. Section B, Biological Sciences 88(5): 347-357. DOI: http://dx.doi.org/10.1007/ BF03046107

Panero, J. L. y V. A. Funk. 2002. Toward a phylogenetic subfamilial classification for the Compositae (Asteraceae). Proceedings of the Biological Society of Washington 115(4): 909-922.

Panero, J. L. y V. A. Funk. 2008. The value of sampling anomalous taxa in phylogenetic studies: Major clades of the Asteraceae revealed. Molecular Phylogenetcs and Evolution 47: 757-782. DOI: http://dx.doi.org/10.1016//j. ympev.2008.02.011

Rabinowitz, S., S. Cairns y T. Dillon. 1986. Seven forms of rarity and their frecuency in the flora of the Brithis Isles. In: Soule, M. E. (ed.). Conservation Biology. The science of scarcity and diversity. Sinauer Associates, Inc. Publishers. Sunderland, USA. Pp. 182-203.

Redonda-Martínez, R. 2006. Delimitación taxonómica de Vernonia Schreb. (Familia Asteraceae, Tribu Vernonieae) y géneros afines en México. Tesis de licenciatura. Facultad 
de Ciencias, Universidad Nacional Autónoma de México. Cd. Mx., México. 76 pp.

Redonda-Martínez, R. 2016. Relaciones filogenéticas de la subtribu Leiboldiinae H. Rob. (Tribu Vernonieae, Familia Asteraceae) con base en caracteres morfológicos. Tesis de doctorado. Instituto de Biología, Universidad Nacional Autónoma de México. Cd. Mx., México. 236 pp.

Redonda-Martínez, R. y J. L. Villaseñor. 2009. Asteraceae, Tribu Vernonieae. In: Medina-Lemos, R., J. G. Sánchez-Ken, A. García-Mendoza, S. Arias-Montes (eds.). Flora del Valle de Tehuacán-Cuicatlán 72: 1-23.

Redonda-Martínez, R. y J. L. Villaseñor. 2011. El género Lepidaploa (Familia Asteraceae, Tribu Vernonieae) en México. Revista Mexicana de Biodiversidad 82(3): 782-797.

Redonda-Martínez, R. y J. L. Villaseñor. 2013. Dos nuevas combinaciones en Vernonieae (Asteraceae) de México. Revista Mexicana de Biodiversidad 84: 983-984. DOI: http://dx.doi.org/10.7550/rmb.34572

Redonda-Martínez, R. y J. L. Villaseñor. 2014. Stramentopappus congestiflorus (Asteraceae: Vernonieae: Leiboldiinae) a new species from Oaxaca, México. Revista Mexicana de Biodiversidad 85: 1-8. DOI: http://dx.doi.org/10.7550/ $\mathrm{rmb} .36354$

Redonda-Martínez R. y E. M. Martínez-Salas. 2015. Lepidonia alba (Asteraceae: Vernonieae: Leiboldiinae) a New Species from the State of Chiapas, Mexico. Systematic Botany 40: 1137-1143. DOI: http://dx.doi. org/10.1600/036364415X690157

Redonda-Martínez, R., J. L. Villaseñor y T. Terrazas. 2012. Trichomes diversity in Vernonieae of Mexico I: Vernonanthura and Vernonia (Vernoniinae). The Journal of the Torrey Botanical Society 139(3): 235-247. DOI: http:// dx.doi.org/10.3159/TORREY-D-11-00069.1

Redonda-Martínez, R., J. L. Villaseñor y T. Terrazas. $2016 \mathrm{a}$. Trichomes diversity in the subtribe Leiboldiinae (Vernonieae, Asteraceae). The Journal of the Torrey Botanical Society 143(3): 298-310. DOI: http://dx.doi.org/10.3159/ TORREY-D-14-00062.1

Redonda-Martínez, R., J. L. Villaseñor y A. Campos-Villanueva. 2016b. A new species of Vernonia (Asteraceae, Vernonieae) from the State of Oaxaca, Mexico. Phytotaxa
282(3): 211-217. DOI: http://dx.doi.org/10.11646/phytotaxa.282.3.4

Robinson, H. 1977. An analysis of the characters and relationships of the Tribes Eupatorieae and Vernonieae (Asteraceae). Systematic Botany 2(3): 199-208. DOI: http://dx.doi.org/10.2307/2418263

Robinson, H. 1980. Re-establishment of the Genus Critoniopsis (Vernonieae: Asteraceae). Phytologia. 46: 437-442. DOI: http://dx.doi.org/10.5962/bhl.part.14736

Robinson, H. 1983. A generic review of the Tribe Liabeae (Asteraceae). Smithsonian Contributions to Botany 54: 1-69. DOI: https://dx.doi.org/10.5479/si.0081024X.54

Robinson, H. 1987a. Studies of the Lepidaploa Complex (Vernonieae: Asteraceae), I: The Genus Stenocephalum Sch. Bip. Proceedings of the Biological Society of Washington 100: 578-583.

Robinson, H. 1987b. Studies in the Lepidaploa Complex (Vernonieae: Asteraceae), III: Two New Genera, Cytocymura and Eirmocephala. Proceedings of the Biological Society of Washington 100: 844-855.

Robinson, H. 1990. Studies in the Lepidaploa Complex (Vernonieae: Asteraceae), VII. The Genus, Lepidaploa. Proceedings of the Biological Society of Washington 103: 464-498.

Robinson, H. 1992. A New Genus Vernonanthura (Vernonieae: Asteraceae). Phytologia 73: 65-76.

Robinson, H. 1993. A review of the genus Critoniopsis in Central and South America (Vernonieae: Asteraceae). Proceedings of the Biological Society of Washington 106: 606-627.

Robinson, H. 1999. Generic and Subtribal Classification of American Vernonieae. Smithsonian Contributions to Botany 89: 1-116. DOI: http://dx.doi.org/10.5962/bhl.title. 103697

Robinson, H. 2007. Tribe Vernonieae. In: Kadereit, J. W. y C. Jeffrey (eds.). The Families and Genera of Vascular Plants. Flowering Plants Eudicots Asterales, Vol. VIII. Springer-Verlag-Wien. Berlin, Germany. Pp. 165-192.

Robinson, H. y C. F. Reed. 1973. A new species of Vernonia from Mexico. Phytologia 27: 52.

Robinson, H. y V. A. Funk. 1987. A phylogenetic analysis of Leiboldia, Lepidonia, and a new genus Stramentopappus 
(Vernonieae: Asteraceae). Botanische Jahrbücher für Systematik, Pflanzengeschichte und Pflanzengeographie 108: 213-228.

Robinson, H. y V. A. Funk. 2011. A new genus, Nothovernonia, from tropical Africa (Asteraceae or Compositae, Vernonieae). Phytokeys 3: 21-34. DOI: http://dx.doi. org/10.3897/ phytokeys.3.1131

Roque, N., D. J. Keil y A. Sussana. 2009. Illustrated glossary of Compositae. In: Funk, V. A., A. Sussana, T. F. Stuessy y R. J. Bayer (eds.). Systematics, Evolution and Biogeography of the Compositae. International Association for Plant Taxonomy (IAPT). Vienna, Austria. Pp. 781-806.

Rzedowski, J. 1978. Vegetación de México. Limusa. México D.F., México. 432 pp.

Rzedowski, J. y G. Calderón de Rzedowski. 1995. Vernonieae. Flora del Bajío y de regiones adyacentes 38: 1-50.

Turner, B. L. 1981. New species and combinations in Vernonia sections Leiboldia and Lepidonia (Asteraceae), with a revisional conspectus of the groups. Brittonia 33(3): 401412. DOI: http://dx.doi.org/10.2307/2806430
Turner, B. L. 1988. New combinations in Mexican Vernonia (sect. Lepidaploa). Phytologia 65: 135-137. DOI: http:// dx.doi.org/10.596/bhl.part.28874

Turner, B. L. 1992. A new species of Vernonia (Asteraceae) from Sonora, Mexico. Phytologia 73: 16-17. DOI: http:// dx.doi.org/10.5962/bhl.part.6216

Turner, B. L. 1997. A new species of Vernonia (s.1.), section Leiboldia, (Asteraceae, Vernonieae) from easternmost Oaxaca, Mexico. Phytologia 82: 275-277.

Turner, B. L. 2007. The comps of Mexico. Chapter 8 Liabeae and Vernonieae. Phytologia Memoirs 12: 35-144.

Villaseñor, J. L., G. Ibarra y D. Ocaña. 1998. Strategies for the Conservation of Asteraceae in Mexico. Conservation Biology 12(5): 1066-1075. DOI: http://dx.doi. org/10.1046/j.1523-1739. 1998.97171.x

Villarreal, J. A. y J. L. Villaseñor. 2004. Familia Compositae, Tribu Tageteae. Flora de Veracruz 135: 1-67. 